\title{
Quantification of Lipoprotein Uptake in Vivo Using Magnetic Particle Imaging and Spectroscopy
}

Staffan Hildebrand, Norbert Löwa, Hendrik Paysen, Raluca M. Fratila, Laia Reverte-Salisa, Thithawat Trakoolwilaiwan, Zheming Niu, Georgios Kasparis, Stephanie Franziska Preuss, Olaf Kosch, Jesus M. de la Fuente, Nguyen Thi Kim Thanh, Frank Wiekhorst, and Alexander Pfeifer*

Cite This: https://dx.doi.org/10.1021/acsnano.0c03229

Read Online

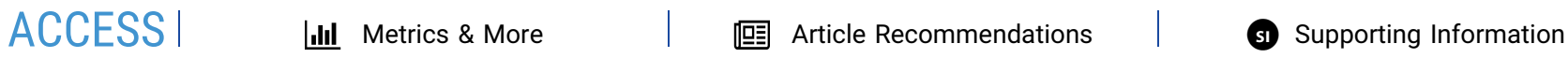

ABSTRACT: Lipids are a major source of energy for most tissues, and lipid uptake and storage is therefore crucial for energy homeostasis. So far, quantification of lipid uptake in vivo has primarily relied on radioactive isotope labeling, exposing human subjects or experimental animals to ionizing radiation. Here, we describe the quantification of in vivo uptake of chylomicrons, the primary carriers of dietary lipids, in metabolically active tissues using magnetic particle imaging (MPI) and

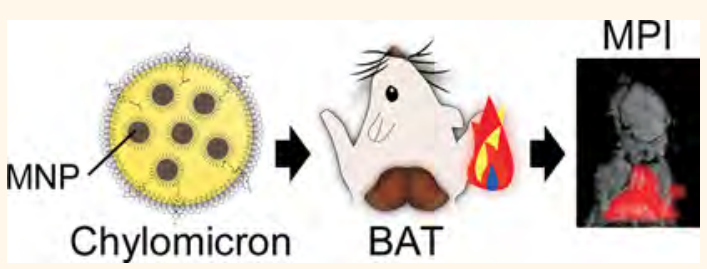
magnetic particle spectroscopy (MPS). We show that loading artificial chylomicrons (ACM) with iron oxide nanoparticles (IONPs) enables rapid and highly sensitive post hoc detection of lipid uptake in situ using MPS. Importantly, by utilizing highly magnetic Zn-doped iron oxide nanoparticles (ZnMNPs), we generated ACM with MPI tracer properties superseding the current gold-standard, Resovist, enabling quantification of lipid uptake from whole-animal scans. We focused on brown adipose tissue (BAT), which dissipates heat and can consume a large part of nutrient lipids, as a model for tightly regulated and inducible lipid uptake. High BAT activity in humans correlates with leanness and improved cardiometabolic health. However, the lack of nonradioactive imaging techniques is an important hurdle for the development of BAT-centered therapies for metabolic diseases such as obesity and type 2 diabetes. Comparison of MPI measurements with iron quantification by inductively coupled plasma mass spectrometry revealed that MPI rivals the performance of this highly sensitive technique. Our results represent radioactivity-free quantification of lipid uptake in metabolically active tissues such as BAT.

KEYWORDS: magnetic particle imaging, brown adipose tissue, BAT, chylomicron, TRL, magnetic particle spectroscopy, lipid uptake

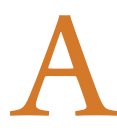

dipose tissues are the major sites of lipid storage. There are two major types of adipose tissue, white adipose tissue (WAT) and brown adipose tissue (BAT). ${ }^{1}$ BAT is an organ that is capable of converting chemical energy into heat by mitochondrial uncoupling. ${ }^{2,3}$ Since the discovery of metabolically active BAT in adult humans in $2007 / 9,^{4-8}$ it has been the subject of intense research due to the possibility of exploiting its thermogenic properties for combating obesity, diabetes, dyslipidemia, and cardiovascular disease. ${ }^{9-11}$ During the process of thermogenesis in BAT, there is a dramatic increase in the demand for energy substrates, and ca. 50-75\% of the food-derived lipids and glucose, respectively, are taken up by activated BAT. ${ }^{12-16}$ Consequently, quantification of lipid uptake in BAT is a highly valuable tool both to measure BAT activity and to develop ways to measure lipid transport and storage. Additionally, safe and noninvasive measurements of BAT activity in humans would be an important prerequisite for the development of antiobesity drugs.
Lipoprotein particles are composed of triglycerides, phospholipids, and cholesterol and are associated with apolipoproteins (ApoA, ApoB, ApoC, and ApoE), which confer functionality and tissue targeting capabilities. ${ }^{17,18}$ Classification of lipoprotein particles is based on particle size and density: high-density lipoprotein particles (HDL), lowdensity lipoprotein particles (LDL), intermediate-density lipoprotein particles (IDL), and very low density lipoprotein particles (VLDL) range from 5 to $100 \mathrm{~nm}$ in diameter, respectively, and take part in lipid transport between the liver and peripheral tissues. Ultra-low-density lipoprotein particles

Received: April 17, 2020

Accepted: December 4, 2020 


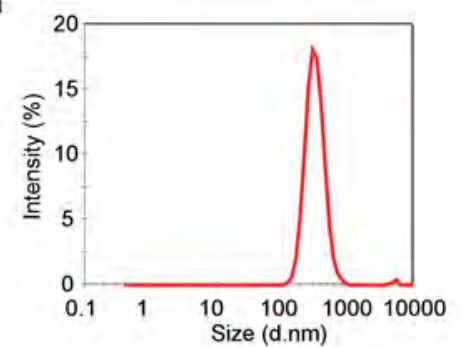

c

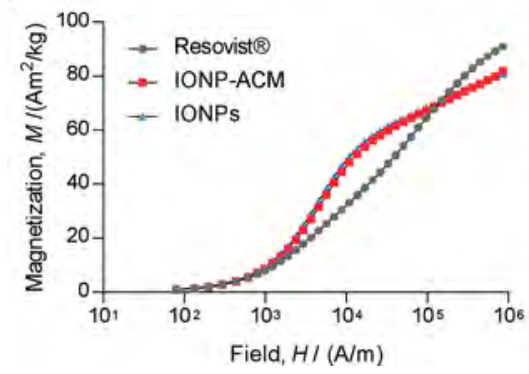

b

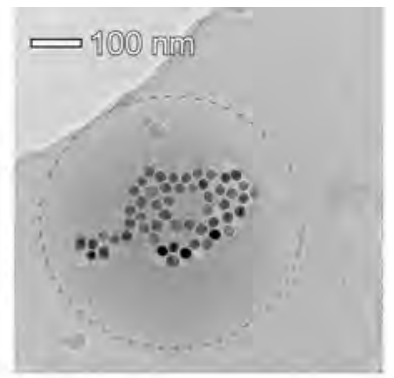

d

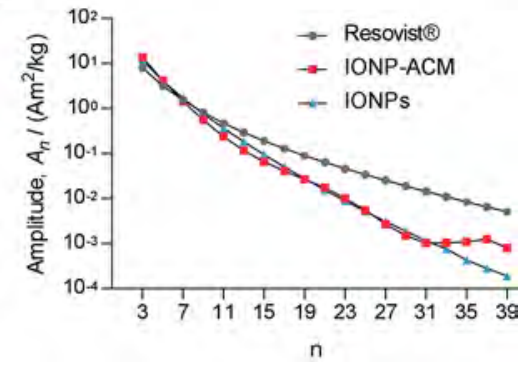

Figure 1. Characterization of IONP-ACM. (a) Size distribution of IONP-ACM as measured by dynamic light scattering (DLS). (b) Representative cryo-TEM image of IONP-loaded ACM. Dashed line outlines the circumference of the ACM. (c) Quasi-static magnetization curves of three batches of IONP-ACM, with Resovist and the IONPs used for loading as reference. (d) Dynamic magnetization measurements of three batches of IONP-ACM, with Resovist and the IONPs used for loading as reference; $n$ represents the harmonic number. IONP-ACM data are presented as means \pm SEM.

(ULDL), also known as triglyceride-rich lipoprotein particles (TRL) or chylomicrons, are between 100 and $1000 \mathrm{~nm}$ in diameter and represent the primary means of transport for dietary lipids between the gut and peripheral tissues. ${ }^{18,19}$ Artificial lipoprotein particles have found a variety of uses in research, including analysis of nutrient uptake and lipid metabolism, diagnostics, and immunotherapy. ${ }^{13,20,21}$ Uptake of chylomicrons in tissues is canonically mediated by lipoprotein lipase at the endothelium, followed by import of the free fatty acids into the tissue. ${ }^{17,22,23}$ Alternatively, lipoprotein particles can undergo whole-particle internalization and transcytosis through the endothelium. ${ }^{13}$ Activated BAT efficiently takes up lipoprotein particles, and the majority of intravenously administered lipoprotein particles are cleared from the serum within minutes. ${ }^{13}$

Currently, the only imaging technique commonly used for detecting nutrient uptake in humans is positron emission tomography-computed tomography (PET-CT), which involves administering a cumulative dose of ionizing radiation of around $30 \mathrm{mSv}$ for a single round of image acquisition. ${ }^{24}$ As this poses a significant health risk for involved subjects, techniques for radiation-free BAT activity measurements are urgently needed. Additionally, the tracers used for metabolic PET-CT imaging, typically $\left[{ }^{18} \mathrm{~F}\right] \mathrm{FDG}$ for glucose or $\left[{ }^{18} \mathrm{~F}\right]$ FTHA for lipids, both have drawbacks that limits their use for assessing BAT activity. First, obese subjects are typically diabetic and exhibit impaired cold-induced glucose uptake (and thereby $\left[{ }^{18} \mathrm{~F}\right] \mathrm{FDG}$ ), but not fatty acids. ${ }^{25}$ Second, it is currently not possible to measure BAT volume directly using $\left[{ }^{18} \mathrm{~F}\right]$ FTHA due to insufficient radioactivity contrast between BAT and WAT. To circumvent these issues, attempts have been made to measure BAT activity with magnetic resonance imaging (MRI). Using dynamic $\mathrm{T} 2 *$-imaging, signal fluctuations that correlate with whole-body cooling can be detected, but so far this technique has been hampered by low specificity and contrast. $^{26}$ MRI has also been used in experimental animal models to detect iron oxide-loaded chylomicrons for semiquantitative measurements. ${ }^{27}$ Other methods for assessing BAT activity have been investigated, such as hyperpolarized ${ }^{13} \mathrm{C}$-MRI imaging to detect metabolite turnover and contrast ultrasound to detect changes in blood flow (although this is limited to superficial tissues). ${ }^{28,29}$

Here, we describe quantification of in vivo lipid uptake using magnetic particle imaging (MPI) and magnetic particle spectroscopy (MPS). The MPI/MPS signal is derived from the nonlinear magnetization curve of superparamagnetic particles, $^{30}$ a phenomenon that does not occur in biological tissues. MPI is an emerging imaging technique that enables not only three-dimensional imaging but also quantification of magnetic nanoparticles (MNPs) in vivo. ${ }^{31,32}$ MPI imaging offers several advantages over current clinically used imaging techniques, such as zero signal tissue attenuation, radiation-free tracers, and high spatial and temporal resolution. ${ }^{32}$ Unlike MRI, the tracers in MPI are the only source for signal, leading to extremely high contrast. Furthermore, MRI-based measurements of MNPs require a reference scan prior to particle administration, and the sensitivity of MRI-based MNP detection has been reported to be more than 20 times lower compared to MPI. ${ }^{33}$ To date, MPI has primarily been used for cell tracking. vascular imaging, and MPI-guided hyperthermia therapy. ${ }^{34-38}$ We show that by using tailored Zn-doped MNPs (ZnMNPs), MNP-loaded chylomicrons enable high-resolution, nonradioactive imaging and quantification of metabolic activity in vivo using MPI. As a model for induced, tissuespecific lipid uptake, we use BAT activation by acute cold exposure and show that MPI detection of internalized MNPs provides an accurate and safe way of detecting alterations in lipid uptake noninvasively. Additionally, we show that the uptake can be quantified ex vivo with high sensitivity in any type of tissue with minimal background noise using MPS (zero-dimensional MPI) using relatively inexpensive and commercially available reagents and equipment. 
a

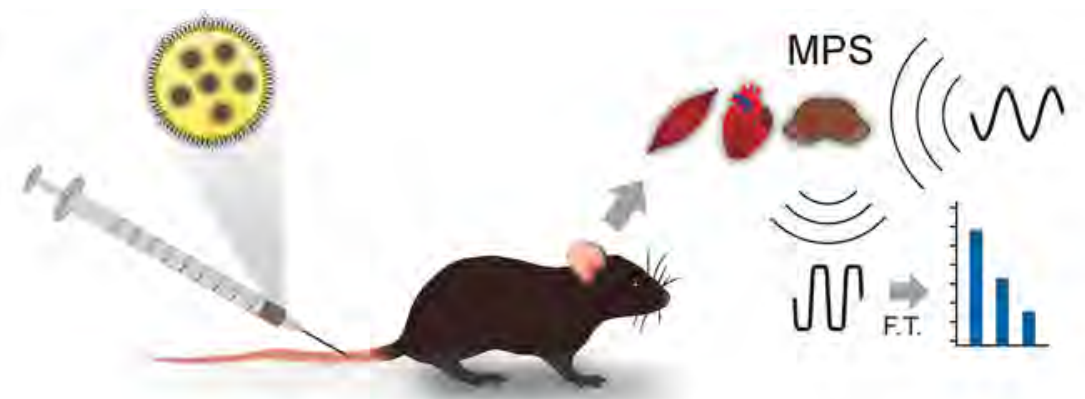

b
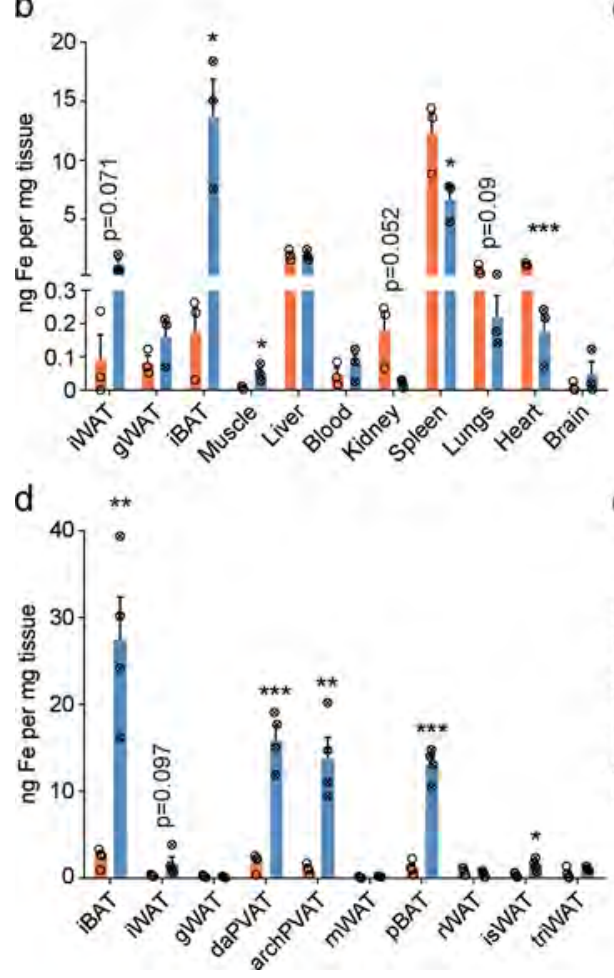

C

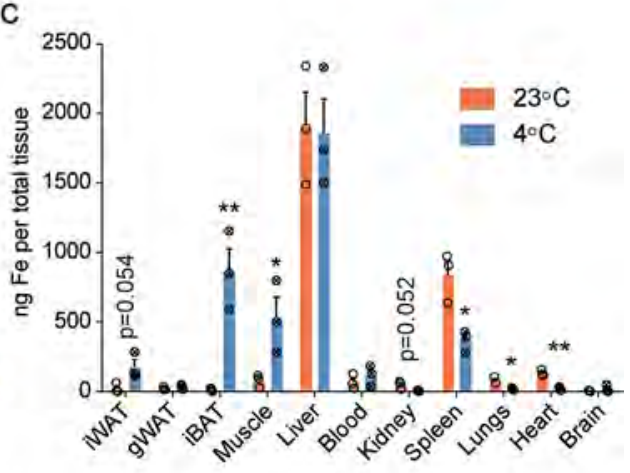

e

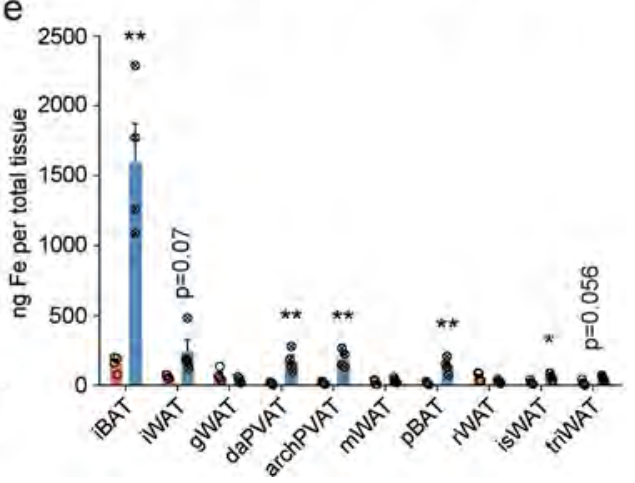

Figure 2. MPS-based quantification of IONP-ACM uptake in tissues after cold exposure. (a) Schematic representation of the experimental setup. F.T.: Fourier transform. (b, c) Specific and total uptake of IONP-ACM in different organs after 20 h of cold-exposure $(n=3$ mice per group). (d, e) Specific and total uptake of IONP-ACM in BAT and WAT depots after $20 \mathrm{~h}$ of cold-exposure ( $n=4$ mice per group). iBAT: interscapular BAT, daPVAT: descending aorta PVAT, archPVAT: aortic arch PVAT. Data are presented as means \pm SEM, $*=p<0.05, * *=$ $p<0.01, * * *=p<0.001$, two-tailed Student's $t$ test, $23{ }^{\circ} \mathrm{C} v s{ }^{\circ} \mathrm{C}$.

\section{RESULTS AND DISCUSSION}

Artificial chylomicrons (ACM) were synthesized by mixing phosphatidylcholine (PC), triglycerides (TG), and cholesterol. To optimize particle size, the TG content was varied, resulting in an optimal size distribution between 100 and $1000 \mathrm{~nm}$ at a TG to PC mass ratio of 16:1 (Figure 1a), with a mean hydrodynamic diameter of $387.2 \pm 13 \mathrm{~nm}$ (Supplementary Figure 1a). For initial experiments using MPS, we loaded ACM with oleic acid-stabilized iron oxide magnetic nanoparticles (IONPs) with a diameter of $20 \mathrm{~nm}$. Incorporation of IONPs in the triglyceride core only slightly decreased the mean hydrodynamic diameter $(338.6 \pm 9 \mathrm{~nm})$, with no discernible effect on polydispersity index or Z-potential (Supplementary Figure $1 \mathrm{~b}, \mathrm{c})$. The potential size distribution bias of IONPloaded ACM was investigated by pulling down highly magnetic IONP-ACM with a permanent magnet and measuring the hydrodynamic size of pulled down ACM and the ones in the nonmagnetic supernatant. We found that highly magnetic IONP-ACM had a slightly higher mean hydrodynamic diameter, but that both fractions showed size distributions characteristic of chylomicrons (Supplementary Figure 1d,e). Embedding of the IONPs in the core of the chylomicrons was corroborated by transmission electron cryomicroscopy (cryoTEM) as well as negative-stain transmission electron microscopy (TEM) and high-angle annular dark-field scanning transmission electron microscopy (HAADF-STEM) (Figure $1 \mathrm{~b}$ and Supplementary Figure 2, respectively). The negativestain TEM and HAADF-STEM images appear to show particles of slightly larger size than the cryo-TEM and hydrodynamic diameter measurements, possibly due to morphological changes caused by the sample preparation (e.g., "flattening" of the particles during sample drying). Importantly, the cryo-TEM analysis showed that IONPs were inside the ACM and not on the surface, indicating stable loading and low likelihood of nanoparticle shedding. Incorporation in ACM did not alter the static and dynamic properties of the IONPs: the quasi-static magnetization curves of IONP-loaded ACM indicate that the individual particles are 
still sufficiently separated, showing no adverse influence of chylomicron synthesis on the magnetization behavior of the IONPs (Figure 1c). Nevertheless, both samples show a nonsaturating magnetization curve at high fields. This linear contribution has been often attributed to surface spin canting in IONPs and is very likely due to a low amount of crystallographic disorder as found in other IONP samples as well. $^{39-41}$ Similar results were obtained by dynamic magnetization measurements using MPS, where no significant change in the spectral magnetic signature was measured for individual IONPs compared to IONP-loaded ACM (Figure 1d).

Recent studies have implicated activated BAT as a major site for postprandial TG clearance. ${ }^{13,14,20,42}$ Therefore, we used acute cold exposure as a means to induce chylomicron uptake in vivo. Cold exposure strongly increases the requirement for intracellular energy substrates to fuel thermogenesis and therefore induces a rapid influx of glucose and lipids from the circulation. ${ }^{4,6,8,13} \mathrm{~A}$ bolus of $5 \mathrm{~mL} / \mathrm{kg}$ IONP-ACM was injected in the tail vein of male C57BL/6-J mice following $20 \mathrm{~h}$ of cold $\left(4{ }^{\circ} \mathrm{C}\right)$ exposure (Figure 2a). MPS measurements of tissue lysates revealed a dramatic increase in Fe uptake in the BAT of cold-exposed animals in terms of Fe uptake both per $\mathrm{mg}$ of tissue $\left(0.2 \pm 0.1 \mathrm{ng} / \mathrm{mg}\left(23^{\circ} \mathrm{C}\right)\right.$ vs $13.7 \pm 3.2 \mathrm{ng} / \mathrm{mg}(4$ $\left.{ }^{\circ} \mathrm{C}\right)$, Figure $\left.2 \mathrm{~b}\right)$ and per whole tissue $\left(13.6 \pm 6.3 \mathrm{ng}\left(23^{\circ} \mathrm{C}\right)\right.$ vs $864.6 \pm 163.1 \mathrm{ng}\left(4^{\circ} \mathrm{C}\right)$, Figure $\left.2 \mathrm{c}\right)$. Compared to visceral WAT, subcutaneous WAT has a large population of inducible brown adipocytes, also known as beige adipocytes, which contribute to whole-body energy homeostasis. ${ }^{1,43,44}$ In line with this, the subcutaneous inguinal WAT depot (iWAT) of cold-exposed mice exhibited a tendency for increased $\mathrm{Fe}$ uptake $\left(0.09 \pm 0.07 \mathrm{ng} / \mathrm{mg}\left(23^{\circ} \mathrm{C}\right)\right.$ vs $1.2 \pm 0.4 \mathrm{ng} / \mathrm{mg}\left(4^{\circ} \mathrm{C}\right)$ for specific uptake, $p=0.0708$, Figure $2 \mathrm{~b}$, and $23.8 \pm 20.2 \mathrm{ng}$ $\left(23^{\circ} \mathrm{C}\right)$ vs $177.9 \pm 53.4 \mathrm{ng}\left(4^{\circ} \mathrm{C}\right)$ for total uptake, $p=0.0541$, Figure 2c). In contrast, no increase in $\mathrm{Fe}$ uptake could be observed in the visceral gonadal WAT depot (gWAT, Figure $2 \mathrm{~b}, \mathrm{c})$. Cold exposure also induces shivering of skeletal muscle, increasing its energy substrate requirements. ${ }^{45,46}$ Accordingly, a significant increase in $\mathrm{Fe}$ uptake was also detected in the quadriceps of cold-exposed mice (Figure 2b). These data demonstrate that ACM uptake in metabolically active tissues is quantifiable by MPS-based measurements.

To evaluate the detection limit of MPS-based measurement of ACM uptake in adipose tissue, we performed a more detailed analysis focusing on minor BAT depots as well as depots of beige fat. Perirenal BAT (pBAT) is a small depot of BAT adjacent to the kidneys. The perivascular adipose tissue (PVAT) surrounding the thoracic aorta is another small fat depot that has been reported to resemble BAT in several studies. $^{47,48} \mathrm{Fe}$ uptake in pBAT and PVAT was increased in cold-exposed animals to 10-fold and 12.2-fold, respectively (Figure 2d). Thus, Fe uptake in the small BAT depots (pBAT and PVAT) was detected by MPS and amounted to similar increases as "classical" interscapular BAT upon cold exposure (Figure 2d). To evaluate ACM uptake in beige adipocytes, we compared visceral WAT, which typically has low numbers of beige adipocytes, to subcutaneous WAT, which more readily undergoes "beiging" upon cold exposure and has a high number of beige adipocytes. Visceral WAT, i.e., gWAT, mesenteric WAT (mWAT), and retroperitoneal WAT (rWAT), did not show any increase in Fe uptake after shortterm cold exposure. On the other hand, the subcutaneous adipose tissue depot triceps-associated WAT (triWAT) showed a tendency for increased Fe uptake $(p=0.056$, Figure 2e, Supplementary Figure 3), and the subcutaneous interscapular WAT (isWAT) depot showed significantly more Fe content after cold exposure (Figure 2e, Supplementary Figure 3). Prolonged cold exposure is well described to induce a strong beige phenotype in WAT. ${ }^{1-3}$ Therefore, to induce a more robust beiging of the WAT, we also exposed mice to 1 week of $4{ }^{\circ} \mathrm{C}$ and injected IONP-ACM. We analyzed Fe uptake in all major WAT depots using MPS and found a significant increase in all measured depots (Supplementary Figure 4). This shows that MPS-based quantification of IONPACM uptake can be used to measure beiging of WAT. Interestingly, this also indicates that activated beige fat clears chylomicrons from the circulation at least partly by wholeparticle uptake. Importantly, these findings demonstrate that MPS-based quantification of lipid uptake has sufficient sensitivity to detect increased lipid uptake in even the smallest BAT depots, as well as in different depots of beige fat. The limit of detection (LOD) for the third-harmonic amplitude $A_{3}$ based MPS quantification was determined to be $2.1 \mathrm{ng}$ of $\mathrm{Fe}$ (Supplementary Figure 5). This enabled accurate quantification of lipid uptake in BAT samples as small as $3 \mathrm{mg}$ even in mice housed at room temperature. Interestingly, the magnetization curve of IONPs showed clearly linear correlation at higher field values, meaning that the spin canting is predominant for this commercial type of particles. Therefore, only a reduced part of the nanoparticular iron will contribute to the MPS signal. Thus, using particles with higher crystallographic order may result in stronger signals and lower detection limits.

To verify that the incorporated IONPs enter brown adipocytes, the mature adipocytes from various adipose tissue depots were separated from the stromal vascular fraction (SVF) containing preadipocytes, endothelial cells, and other nonadipocytes. In both BAT and iWAT, Fe uptake was significantly increased in the mature adipocyte fraction compared to the SVF fraction upon cold exposure (5.5 \pm 1.0-fold and $1.8 \pm 0.1$-fold, respectively (Figure 3a,b), but not in animals kept at ambient temperature. In gWAT, no significant difference in $\mathrm{Fe}$ uptake between mature adipocytes and SVF could be observed (Figure 3c). Additionally, TEM and STEM images of BAT samples from cold-exposed, IONPACM-injected mice showed both IONP-ACM apparently undergoing endocytosis in the endothelium and deposition of the IONPs specifically in brown adipocytes (Figure $3 \mathrm{~d}$ and Supplementary Figure 6). The latter was confirmed by energydispersive X-ray spectroscopy (EDX) analysis of BAT tissue sections (Supplementary Figure 7). IONP deposition in the BAT of cold-exposed mice was also confirmed with Prussian Blue staining, which showed bright blue signal in the BAT of mice exposed to $4{ }^{\circ} \mathrm{C}$, but not in those exposed to $23{ }^{\circ} \mathrm{C}$ (Figure 3e). Therefore, we conclude that $\mathrm{Fe}$ content as measured by MPS and MPI after IONP-ACM injection represents true chylomicron uptake in BAT.

To examine the feasibility of using MPI together with the MNP-loaded ACM for measuring lipid uptake and metabolic activity in vivo, the suitability of ACM as MPI tracers was assessed. The harmonic spectra of the IONP-ACM used in the experiments above showed low amplitudes in the higher harmonics compared to Resovist and were thus considered inadequate for MPI. To improve the harmonic spectra of the ACM and thus MPI signal, we focused on incorporating nanoparticles with higher magnetic saturation. Zn-doping of IONPs has previously been shown to increase saturation 
a
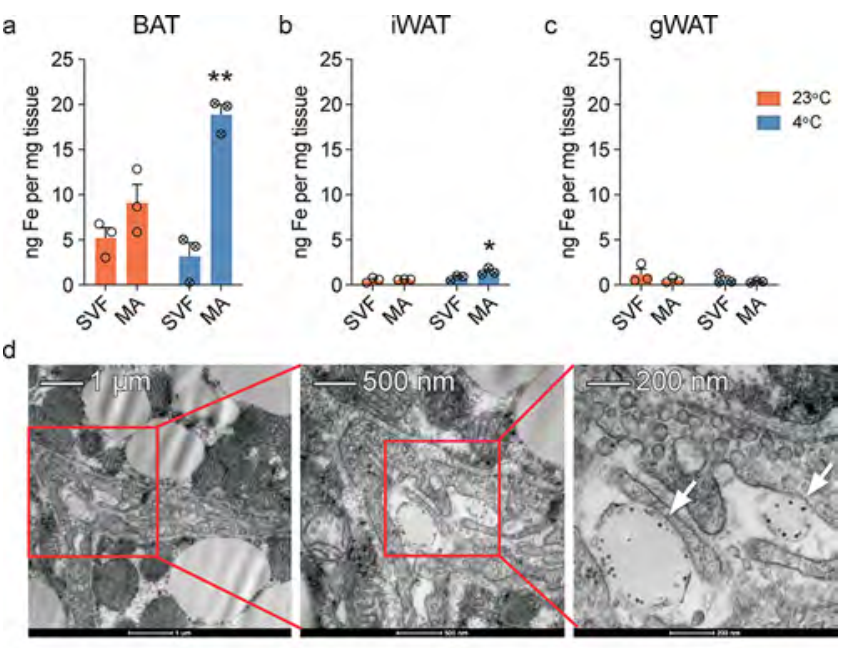

e

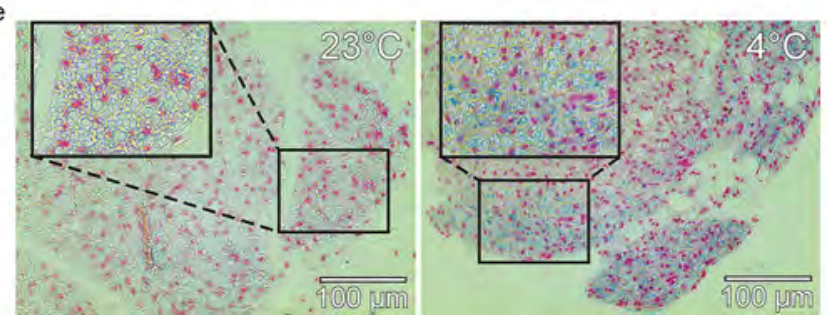

Figure 3. IONP-ACM are specifically taken up by the brown adipocytes in BAT. $(\mathrm{a}-\mathrm{c})$ Fe uptake in SVF and MA in BAT, iWAT, and gWAT after exposure to either 23 or $4{ }^{\circ} \mathrm{C}(n=3$ mice per group). (d) TEM images showing uptake of IONP-ACM (arrows) in the capillaries of BAT. (e) Representative images of Prussian Blue/Pararosaniline-stained BAT from mice injected with IONP-ACM after exposure to 23 or $4{ }^{\circ} \mathrm{C}$. Data are presented as means \pm SEM. $*=p<0.05$, two-tailed Student's $t$ test, SVF $v$ s MA.

magnetization. ${ }^{49}$ Therefore, we focused on ZnMNPs and incorporated these into ACM. The ZnMNPs used here had a mean diameter of $10 \pm 2 \mathrm{~nm}$ (as measured by TEM) and a saturation magnetization of $110 \mathrm{emu} / \mathrm{g}$. The resulting ZnMNP-ACM showed high signal amplitudes and a rich harmonic spectrum comparable to that of Resovist (Figure 4a). As the signal amplitudes in the harmonic spectrum strongly depend on the magnetization properties of the particles, we also compared the magnetization curves of Resovist with that of the ZnMNP-ACM. This showed that the ZnMNP-ACM had a considerably higher saturation magnetization than Resovist (Figure 4b). This indicates that, unlike IONP-ACM, the magnetic properties of ZnMNP-ACM make them well suited for MPI applications. The ZnMNP-ACM had a mean diameter of $309.3 \pm 13.2 \mathrm{~nm}$ (Supplementary Figure 8a), with a similar size distribution to that of the IONP-ACM (Supplementary Figure $8 \mathrm{~b}$ ). Incorporation of ZnMNPs in the ACM was confirmed by HAADF-STEM and EDX (Supplementary Figure 8c).

A systematic MPS evaluation of the ZnMNP-ACM as MPI tracer was then performed with Resovist as reference. Importantly, serial dilution of ZnMNP-ACM showed that the MPS spectrum of ZnMNP-ACM scales linearly with concentration (Figure 4c,d), which is necessary for quantitative MPI. Additionally, encapsulation of the ZnMNPs in ACM rendered their magnetic properties extremely insensitive to the surrounding environment, unlike the current gold-standard tracer for MPI, Resovist (Figure 4e). To compare the spatial resolution performance of Resovist and ZnMNP-ACM, we also measured the full width at half-maximum (fwhm) of point-like phantoms, which showed slightly increased fwhm for ZnMNPACM (Supplementary Figure 9). These data indicate that ZnMNP-ACM are well suited for use as MPI tracers.

To demonstrate the performance of ZnMNP-ACM as MPI tracers, $\mathrm{ZnMNP}$-ACM were administered to mice, which were then analyzed by whole-animal MPI scans (see Supplementary Figure 10 for workflow). As shown in Figure 5, MPI enabled clear visualization of lipid uptake in active BAT. For comparison, control mice were injected with Resovist with or without prior cold exposure. In contrast to ZnMNP-ACMinjected mice, no MPI signal could be detected in the interscapular area of Resovist-injected mice (Figure 5b). MPSbased quantification of Resovist uptake in isolated organs showed no uptake in BAT, iWAT, or gWAT (Supplementary Figure 11). Quantification of ZnMNP content in the interscapular area of mice injected with ZnMNP-ACM showed a significant increase upon cold exposure (8.7 \pm 1.0-fold, Figure 5c,d).

After measuring tissue uptake of ZnMNP-ACM by wholebody MPI scans, tissues were extracted from the mice and $\mathrm{Fe}$ content was quantified again ex vivo using both MPI (Figure $6 \mathrm{a}-\mathrm{c})$ and MPS. MPI quantification of the Fe content in extracted BAT showed a similar increase in Fe content as the whole-body scans $(10.4 \pm 1.5$-fold, Figure $6 \mathrm{~b})$. The imaging of individual organs also confirmed that no signal was emanating from organs proximal to the BAT, such as the lungs or heart (Figure 6c). Although we saw a tendency for increased uptake in iWAT in our MPS measurements, we were not able to detect any differences with MPI (Figure 6c), possibly due to slightly lower sensitivity when compared to ex vivo MPS. We did not detect any differences in BAT uptake between mice housed at $23{ }^{\circ} \mathrm{C}$ compared to mice housed in thermoneutral conditions $\left(30^{\circ} \mathrm{C}\right.$, Supplementary Figure 12$)$. This is in good agreement with data on radioactively labeled artificial chylomicrons, which are taken up by BAT through wholeparticle endocytosis mainly upon cold exposure. ${ }^{14}$ The results from MPI- and MPS-based measurement of ZnMNP content correlated strongly $\left(r^{2}>0.99\right)$, underlining that the selected algorithm and the parameters for image reconstruction do not adversely affect the quantitative information contained in the raw data of the MPI measurement (Supplementary Figure 13).

As the injected ACM to a large extent end up in the liver, we performed a long-term elimination study to investigate hepatic clearance of the ZnMNP-ACM. This showed a significant decrease of MNP content over time, with less than $20 \%$ of the MPS signal remaining after 4 weeks (Supplementary Figure 14). To verify that the ZnMNP-ACM uptake measured by MPI is indeed due to uptake of Zn-MNP-loaded ACM and not free $\mathrm{ZnMNPs}$, we also injected cold-exposed and control mice with free ZnMNPs. We could not detect any uptake of ZnMNPs in BAT using MPS in either group, suggesting that our MPI results represent bona fide ACM uptake (Supplementary Figure 15). Although both zinc and iron are widely considered to be biocompatible materials with minimal safety concerns in the concentration range used in this study, ${ }^{50,51}$ we also investigated the effect of both ZnMNP-ACM and Resovist on brown adipocytes in vitro. We could not detect any significant effect of the nanoparticles on brown fat differentiation, cellular toxicity, or inflammatory cytokine expression (Supplementary Figures 16 and 17). The in vivo toxicity of zinc-doped iron oxide nanoparticles has been studied after 


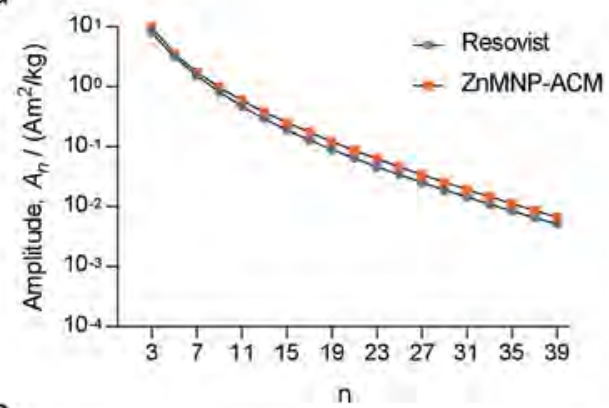

C

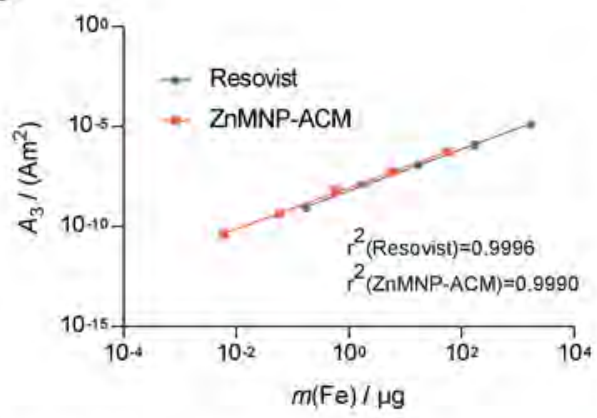

e

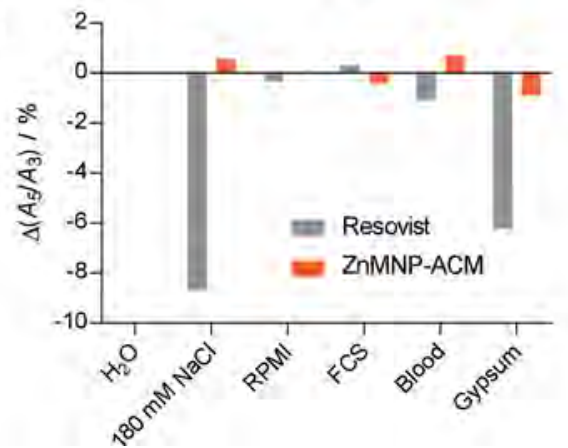

b

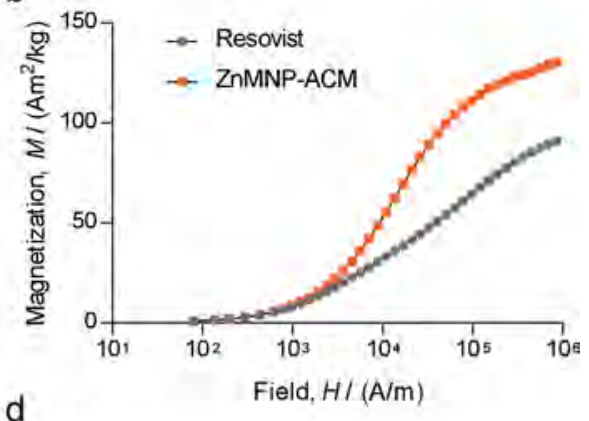

d

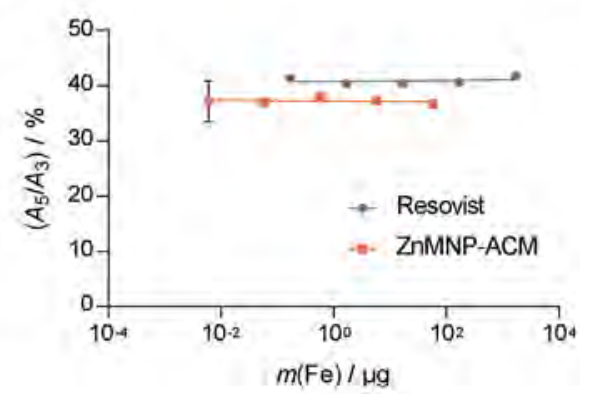

Figure 4. MPS-based evaluation of ZnMNP-ACM as MPI tracer. (a) MPS amplitude spectrum of ZnMNP-ACM and Resovist. (b) Magnetization curves of ZnMNP-ACM and Resovist. (c) MPS signal amplitude after serial dilution parametrized by A3. (d) MPS spectrum shape after serial dilution parametrized by the harmonic ratio A5/A3. (e) Change of MPS signal shape (A5/A3) in different biological environments. Data are presented as means \pm SD of 20 repeated measurements. RPMI: Roswell Park Memorial Institute medium, FCS: fetal calf serum.

intragastric administration, with no observed adverse effects. ${ }^{52}$ Additionally, intravenous administration of zinc oxide nanoparticles led to adverse effects in rats starting at $5 \mathrm{mg} / \mathrm{kg}^{53}$ The zinc content in the MNPs used in this study is $\sim 6.5$ times lower than the iron content. This results in a zinc dose of approximately $300 \mu \mathrm{g} / \mathrm{kg}$ for the ZnMNP-ACM-injected mice, more than 15 times less than previously observed toxic doses of zinc oxide nanoparticles. Therefore, we do not expect the zinc doping to be a limiting factor in terms of toxicity of the ZnMNP-ACM.

Finally, to validate the quantitative performance of our MPI measurements, we compared our results with inductively coupled plasma mass spectrometry (ICP-MS) measurements of the same tissue samples. This showed a relatively small (1.46 \pm 0.15 -fold) but significant increase in $\mathrm{Fe}$ concentration in BAT from cold-exposed mice (Figure $7 \mathrm{a}$ ) and a similar increase $(1.52 \pm 0.23$-fold, $p=0.0644)$ in $\mathrm{Zn}$ concentration (Figure $7 \mathrm{~b}$ ). The concentrations of iron and zinc in coldexposed mice were still within the physiological ranges, indicating low risk for toxicity. Liver and spleen $\mathrm{Fe}$ and $\mathrm{Zn}$ concentrations were also both within physiological ranges (Figure $7 \mathrm{a}, \mathrm{b}) .{ }^{54,55}$ Comparison of our MPI-based (magnetic)
Fe uptake measurements on the ppm scale revealed that the increased concentration of Fe in BAT after cold exposure is not significantly different when measured by MPI or ICP-MS (Figure 7c, Supplementary Figure 18). This shows that MPIbased measurement of Fe uptake in BAT is comparable in sensitivity to ICP-MS, albeit with much higher specificity due to the fact that MPI measures only magnetic iron, and not endogenous iron present in the tissues. Importantly, these data show that quantification of ZnMNP-ACM-derived iron using MPI is comparable in performance with the current gold standard in iron quantification in tissue lysates.

The main limitation of the MPI-based quantification of lipid uptake observed in this study was related to the small field of view (FOV) of current preclinical MPI scanners. Using the highest gradient strength to achieve a highly spatially resolved image, the FOV is limited to around $2 \times 2 \times 1 \mathrm{~cm}^{3}$. A multipatch acquisition scheme was used to increase the total FOV, but due to signals generated from nanoparticles outside the FOV, imaging artifacts at the edges of each individual reconstruction were present. While we partly corrected for this by "overscanning" the field-free point trajectory ${ }^{56}$ to remove imaging artifacts from outside the FOV, we believe that these 
a

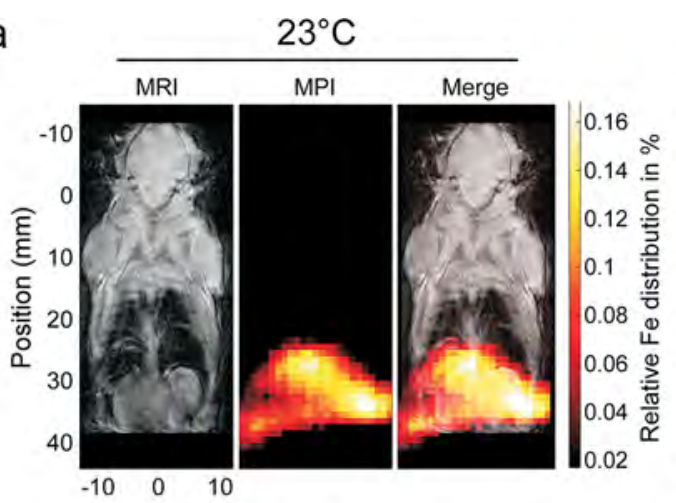

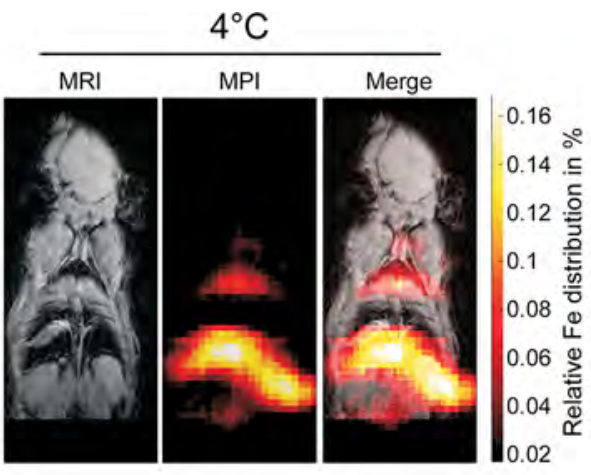

b

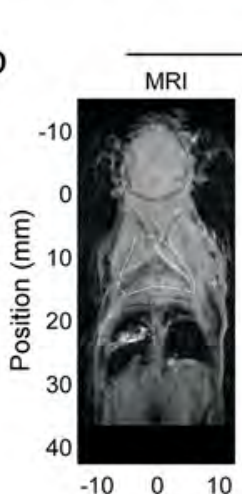

$23^{\circ} \mathrm{C}$

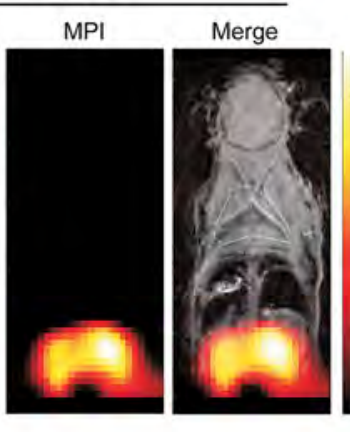

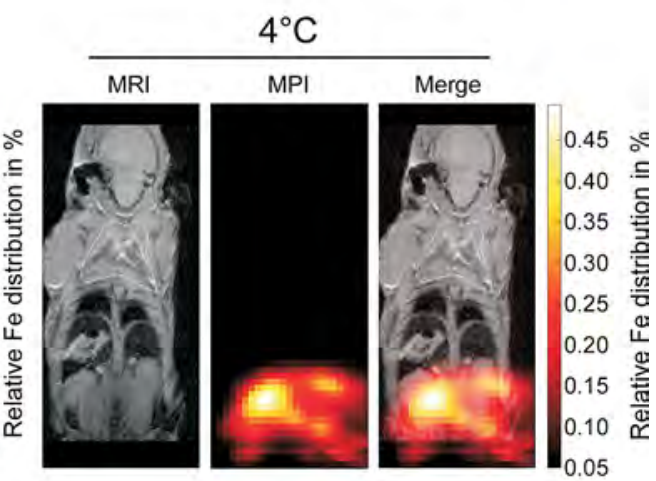

C

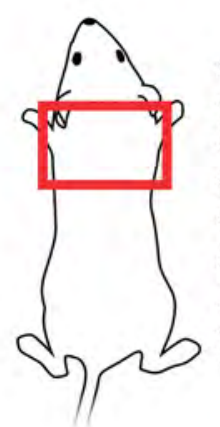

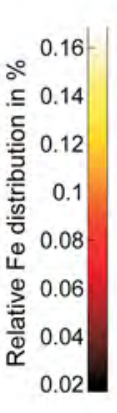

(1) d

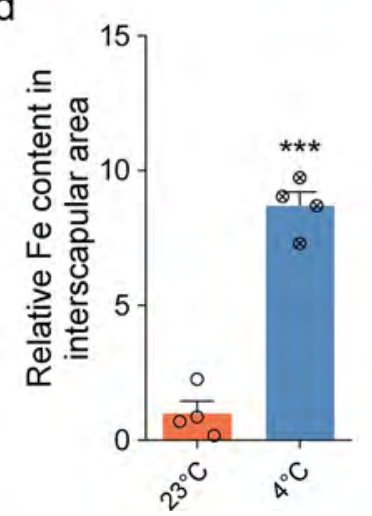

Figure 5. MPI-based quantification of ZnMNP-ACM uptake in BAT after cold exposure. (a) Representative whole-body MRI and MPI images of mice injected with ZnMNP-ACM after exposure to either 23 or $4{ }^{\circ} \mathrm{C}$. (b) Representative whole-body MRI and MPI images of mice injected with Resovist after exposure to either 23 or $4{ }^{\circ} \mathrm{C}$. (c) Representative MPI images of the interscapular area (red box) with MRI overlay. (d) ZnMNP-ACM uptake in BAT after $20 \mathrm{~h}$ of cold-exposure measured from whole-animal MPI scans $(n=4$ mice per group). Data are presented as means \pm SEM relative to uptake at $23{ }^{\circ} \mathrm{C}$ in each tissue, $* *=p<0.01, * * *=p<0.001$, two-tailed Student's $t$ test, $23{ }^{\circ} \mathrm{C} v s$ $4{ }^{\circ} \mathrm{C}$.

problems can be resolved better by a different system function acquisition scheme using focus fields. ${ }^{57}$ While the resolution achieved using ZnMNP-ACM was sufficient to accurately resolve BAT using MPI, it is likely that improvements could be made by further tracer optimization, as other studies have demonstrated MPI tracers with an even larger relative increase in fwhm compared to Resovist. ${ }^{58,59}$ Another potential limitation in regard to absolute quantification of iron uptake using MPI is the possibility of nanoparticle aggregation after uptake, which may affect the MPI signal. Although the ZnMNP-ACM do not show any altered magnetic behavior in different biological environments, we cannot exclude that intracellular processing of the ZnMNP-ACM results in some degree of nanoparticle aggregation. However, since the MPI- based quantification does not yield significantly different results compared to ICP-MS, one would not expect this effect to have a significant impact on the measurements. Given the recent advancement of the development of human MPI scanners, ${ }^{60}$ investigating lipid uptake and BAT metabolic activity in human subjects without significant exposure to radiation may be possible.

\section{CONCLUSIONS}

Current strategies for the quantification of in vivo lipid uptake rely on radioactive labeling of fatty acids, cholesterol, or other components of the lipoprotein particles. ${ }^{13,14,42,45,61-63}$ While this method has benefits in terms of versatility and by being a bona fide measure of lipid uptake, there are clear practical and 
a

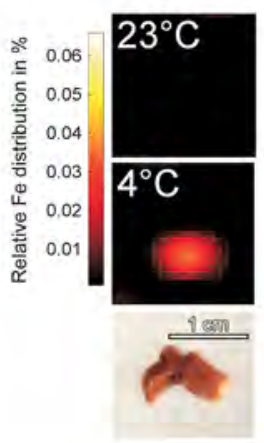

C

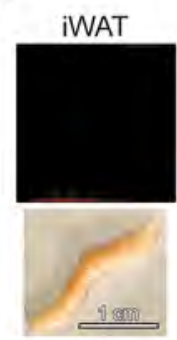

b
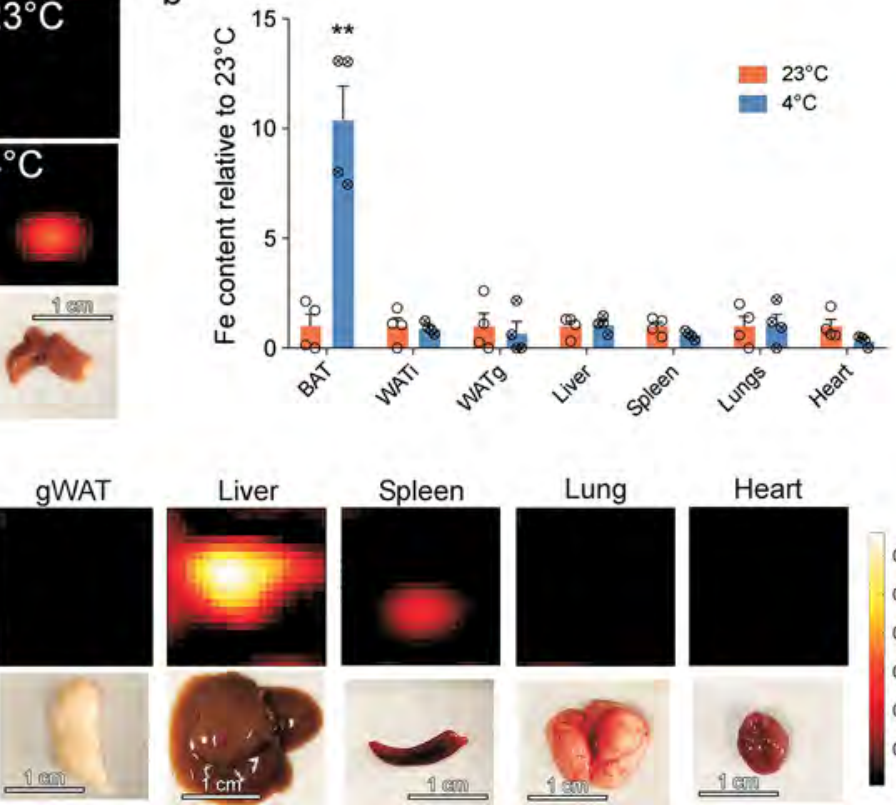

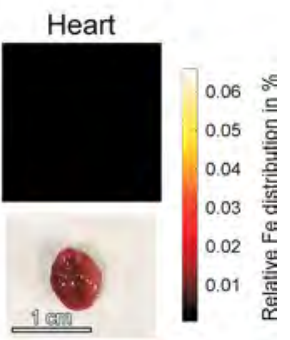

Figure 6. MPI-based quantification of ZnMNP-ACM uptake in isolated organs after cold exposure. (a) Representative MPI images of excised BAT, with representative photograph of BAT at the same scale. (b) ZnMNP-ACM uptake in excised tissues after $20 \mathrm{~h}$ cold-exposure measured from single-organ MPI scans $(n=4$ mice per group). Data are shown as fold-change over Fe content in each organ of the mice housed at $23{ }^{\circ} \mathrm{C}$. (c) Representative MPI images of isolated organs from cold-exposed mice injected with ZnMNP-ACM. Representative photographs of the organs imaged are shown below each MPI image. Data are presented as means \pm SEM relative to uptake at $23{ }^{\circ} \mathrm{C}$ in each tissue, ${ }^{* *}=p<0.01,{ }^{* * *}=p<0.001$, two-tailed Student's $t$ test, $23{ }^{\circ} \mathrm{C} v s{ }^{\circ} \mathrm{C}$.
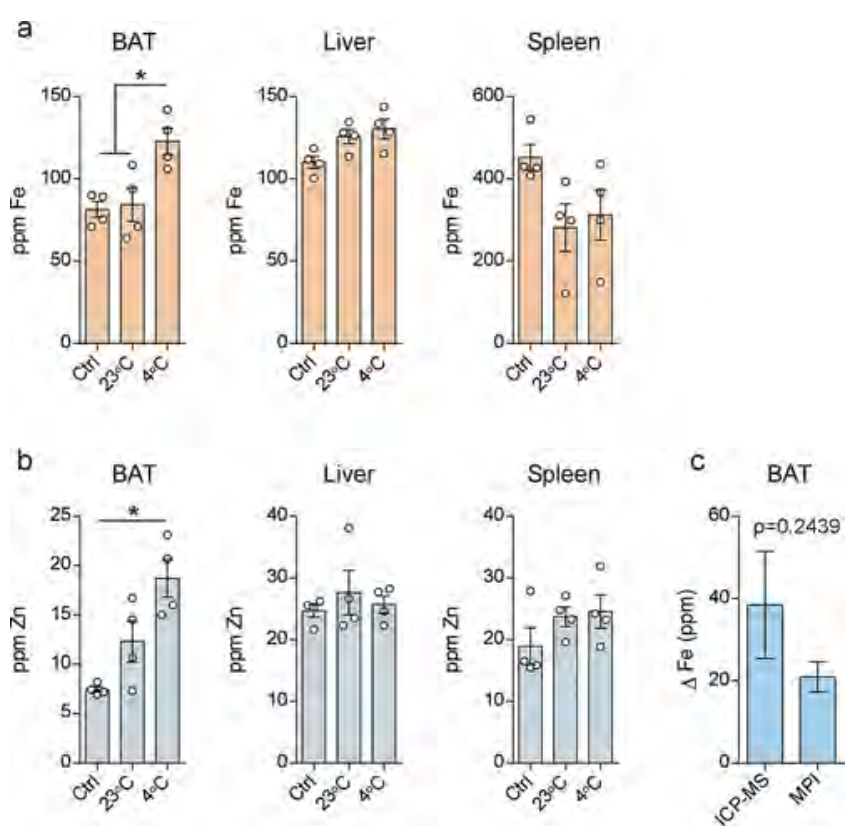

Figure 7. ICP-MS-based quantification of ZnMNP-ACM uptake in BAT after cold exposure. (a) ICP-MS measurements of Fe concentration in tissue samples from ZnMNP-ACM-injected mice ( $n=4$ mice per group). (b) ICP-MS measurements of $\mathrm{Zn}$ concentration in tissue samples from ZnMNP-ACM-injected mice. (c) Comparison between increase in Fe content in BAT upon cold exposure and ZnMNP-ACM injection, as measured by ICP-MS and MPI ( $n=4$ mice per group). Ctrl: control mice, not injected with ZnMNP-ACM. Data are presented as means \pm SEM, $*=p<0.05$, two-tailed Student's $t$ test, $23{ }^{\circ} \mathrm{C} v s 4^{\circ} \mathrm{C}$ or ICP-MS vs MPI.

safety-related drawbacks. Apart from the health concerns inherent in working with radioactive substances, the most common radioisotope for metabolic imaging is ${ }^{18} \mathrm{~F}$, which has a half-life of $110 \mathrm{~min}$, meaning on-site cyclotrons are often required. In this work, we have demonstrated a safe, fast, and highly sensitive technique for measuring chylomicron uptake in metabolically active tissues using IONP-loaded chylomicrons and MPS. Furthermore, we have shown that chylomicrons loaded with strongly magnetic ZnMNPs are highly suitable for MPI imaging, enabling quantification of MNP uptake with similar sensitivity to gold-standard ex vivo methods such as ICP-MS. Taken together, using these tracers, we were able to noninvasively image and quantify chylomicron uptake in metabolically active tissues with great accuracy without relying on radioactive isotope labeling.

\section{METHODS}

Synthesis of Artificial MNP-Loaded Chylomicrons. Artificial chylomicrons were synthesized by mixing cholesterol (Sigma-Aldrich, St. Louis, MO, USA), phosphatidylcholine (Avanti Polar Lipids, Alabaster, AL, USA), and triglycerides (soy bean oil, Sigma-Aldrich) in a 1:1:16 ratio, unless otherwise described. The mixture was dissolved in chloroform (Sigma-Aldrich), and $500 \mu \mathrm{g}$ of superparamagnetic IONPs (Sigma-Aldrich, product no. 900088) or ZnMNPs (see below) were added. The mixture was then placed in a rotary evaporator with a heated water bath $\left(60^{\circ} \mathrm{C}\right)$ at 75 mbar until all chloroform had evaporated. The resulting lipid film was mixed with $1 \mathrm{~mL}$ of phosphate-buffered saline and sonicated with a probe sonicator at maximum power for $10 \mathrm{~min}$ with intermittent cooling on ice. Before use, the MNP-ACM suspension was filtered through a 1 $\mu \mathrm{m}$ poly(ether sulfone) (PES) syringe filter (GE Healthcare Life Sciences, Chicago, IL, USA) and immediately characterized using DLS (ZetaSizer 90S, Malvern Panalytical, Malvern, UK).

Zn-Doped Iron Oxide Nanoparticles. The synthesis of zinc ferrite nanocrystals was prepared by incorporating a zinc precursor in a triethylene glycol and iron acetylacetonate mixture as described previously in a high-temperature and high-pressure reaction. ${ }^{64}$ 
ZnMNP Surface Functionalization. To render the nanoparticles hydrophobic, oleic acid was used to coat the surface. Briefly, oleic acid $(0.5 \mathrm{~mL})$ was dissolved in hexane $(5 \mathrm{~mL})$ under magnetic stirring. Then crude nanoparticle suspension $(0.5 \mathrm{~mL})$ was added, and the reaction was allowed to proceed overnight at room temperature. The black hexane layer was isolated and washed three times with water to remove triethylene glycol and once with ethanol to remove excess oleic acid. The oleic acid-coated nanoparticles were collected by magnetic decantation and redispersed in hexane. The surface coating was assessed by infrared spectroscopy using an ATR-FTIR instrument (Spectrum 100, PerkinElmer) as shown in Supplementary Figure 19.

ZnMNP Material Characterization. The size and morphology of the crystallites was assessed by TEM by measuring at least 300 particles using the image processing software ImageJ. The spherical ZnMNPs have an average size of $10 \pm 2 \mathrm{~nm}$. The magnetic properties were measured on a superconducting quantum interference vibrating sample vibrometer (SQUID-VSM, MPMS 3, Quantum Design, USA).

Analysis of MNP-ACM Uptake in Vivo. Wild-type male 8-10week-old C57Bl/6-J mice were purchased from Janvier and randomly divided into two groups (cold exposure or control). The mice were housed at either 23 or $4{ }^{\circ} \mathrm{C}$ for $20 \mathrm{~h}$ or 1 week prior to injection, with free access to chow and water. A bolus of $5 \mathrm{~mL} / \mathrm{kg}$ of MNP-ACM suspension was injected in the tail vein, immediately followed by an additional $4 \mathrm{~h}$ at either 30,23 , or $4{ }^{\circ} \mathrm{C}$, after which the animals were sacrificed. The investigators could not be blinded to the treatment groups due to the obvious differences in temperatures. All animal experiments were approved by the Landesamt für Natur, Umwelt and Verbraucherschutz, Nordrhein-Westfalen, Germany. The governmental approval number is 84-02.04.2016.A171. A total number of 65 mice were used in this study.

MPS Measurements of Tissue Lysates. Tissues were extracted with ceramic-coated surgical tools and digested overnight with Proteinase K (Roche, Basel, Switzerland). Digested tissues were homogenized by adding 5-10 glass beads (Sigma-Aldrich) followed by rapid shaking for $20 \mathrm{~s}$ in CapMix (3M ESPE, Seefeld, Germany). A $100 \mu \mathrm{L}$ portion of the homogenized lysate was transferred to a PCR tube (Applied Biosystems, Foster City, CA, USA, MicroAmp fast reaction tube with cap) and measured in the MPS system as described above. For measurements of MNP uptake in different tissue compartments, BAT, iWAT, and gWAT were digested with collagenase, and mature adipocytes were separated from other cells by centrifugation. Prior to quantification a serial dilution of a reference sample was measured by MPS to determine the MNP-specific detection sensitivity from the slope of the calibration curve, i.e., the normalized third-harmonic amplitude, $A_{3} *=A_{3} / m(\mathrm{Fe})$. The iron content of the individual tissue sample was determined from the measured $A_{3}$ normalized to $A_{3}{ }^{*}$ of the reference. For larger sample sizes a correction of the signal amplitude was necessary taking the spatial dependence of the coil sensitivity into account. ${ }^{65}$ To further reduce the quantification uncertainty, a subsequent correction of the MNP-specific amplitude $A_{3}{ }^{*}$ was performed as previously described for the quantification of MNPs in biological environments and cells. ${ }^{66,67}$

MPI Measurements. A preclinical MPI scanner (25/20 FF, Bruker BioSpin, Billerica, MA, USA) was used for all MPI measurements. All MPI measurements were performed post mortem. A 3D signal excitation was performed in the $x / y / z$-direction, with slightly different frequencies of $\sim 24.51 / 26.04 / 25.25 \mathrm{kHz}$ and excitation amplitudes of $12 \mathrm{mT}$. Spatial encoding was achieved by static magnetic gradient fields with gradient strengths of $1.25 / 1.25 / 2.5 \mathrm{~T} / \mathrm{m}$. Magnetic response signals, generated by the magnetic nanoparticles, were acquired with a gradiometric receive-only coil. ${ }^{68}$ The acquired signals were used for image reconstruction of the particle distribution based on the system function approach, ${ }^{69,70}$ after background correction by subtraction of empty scanner measurements and truncation based on the signal-to-noise ratio.

A system function was acquired by measurements of a small reference sample of MNPs at multiple positions all over the FOV. Due to the small FOV size of MPI, each mouse was measured at four different positions for $10 \mathrm{~s}$ (500 averages), and particle distributions were reconstructed individually. The resulting images were joined in one final image covering a FOV of $80 \times 25 \times 19 \mathrm{~mm}^{3}$ with a voxel size of $1 \mathrm{~mm}^{3}$. To remove noise from the final images, a threshold intensity was determined by reconstructions of empty scanner measurements. Detailed lists of all MPI-related reconstruction parameters for mice and organs are given in Supplementary Table 1 and 2, respectively. For Fe quantification using MPI data, magnetic iron mass was correlated to voxel intensities based on the system function acquired with a reference sample with known mass. For color-coding of MPI images, the intensity signal was rescaled from arbitrary units of voxel intensity to relative Fe distribution in percent by setting the sum of all voxel intensities in the images to one.

MRI Measurements. Anatomical reference scans were acquired using a preclinical $1 \mathrm{~T}$ ICON MRI system (Bruker BioSpin) equipped with a whole-body receive coil. The same animal bed was used for the MRI and MPI scans without moving the mice between each acquisition. Coronal slices of each mouse were acquired using a FLASH sequence. A detailed list of all MRI-related imaging parameters is given in Supplementary Table 3. Image overlays of MRI and MPI images were performed with the help of fiducial markers. Co-registration of the MPI and MRI images was performed the following way: First, MPI measurements of fiducial markers containing MNPs were performed to get reference information on which location of the MPI-FOV corresponds to which location on the animal bed. Before the MRI measurements of each mouse, a photo was taken. An additional fiducial marker, containing only water, was positioned below the animal bed to be able to overlay the MRI image with the photo. Combined with the information obtained from the MPI measurements of the fiducial markers, the MPI and MRI images can be overlaid. For MPI-based uptake quantification in whole-body scans, the ROI (region of interest) for each mouse was selected by identifying the location of the interscapular BAT in the MRI scans, based on the surrounding anatomy.

Quasi-static Magnetic Characterization. The quasi-static magnetization $M$ of the samples as a function of applied field strength $H$ (up to $4000 \mathrm{kA} / \mathrm{m}$ ) was measured at room temperature using a superconducting quantum interference device (SQUID)-based susceptometer (QD-MPMS-XL-5, Quantum Design, USA). A detailed description of the magnetization measurement procedure is found elsewhere. ${ }^{71}$ Here, we used a polycarbonate capsule as a sample container and a clear straw as a sample holder. The background signal caused by the empty capsule and diamagnetic susceptibility of the dispersion medium was subtracted from the measured signal. The presented magnetization curve results from normalizing the magnetic moment of the sample to its iron content and is given in $\mathrm{Am}^{2}$ per kilogram iron. Therefore, the iron content of the respective sample was determined using a phenanthroline-based iron assay described elsewhere. ${ }^{72}$ Measurement data were fitted by the weighted sum of Langevin functions assuming a log-normal distribution of noninteracting magnetic moments.

Dynamic Magnetic Characterization. The dynamic magnetic characterization of MNP was performed by magnetic particle spectroscopy using a commercial MPS device (MPS-3, Bruker Biospin, GER) ${ }^{65}$ Based on the same physical principle as magnetic particle imaging, waiving any spatial encoding, MPS allows for preevaluation of the MPI performance of MNP. For the measurement a PCR tube (Applied Biosystems, MicroAmp fast reaction tube with cap) containing a sample volume of up to $100 \mu \mathrm{L}$ was placed in the MPS system. By exposing the sample to a sinusoidal magnetic field (amplitude $B_{\mathrm{p}}$ up to $25 \mathrm{mT}, f_{0}$ of $25 \mathrm{kHz}$ ) the induced magnetization is measured by gradiometric pick-up coils. Fourier transformation of the measured time signal results in spectral components showing distinct amplitudes at odd multiples of the drive frequency $f_{0}$ evoked by the characteristic nonlinear magnetization behavior of MNP. In this study all measurements were performed at $37{ }^{\circ} \mathrm{C}$. The LOD of the third-harmonic amplitude $A_{3}$ was determined according to guidance of the International Union of Pure and Applied Chemistry (IUPAC) as $3 \times$ the standard error of the regression $y$-intercepts (residual standard deviation) in the calibration curve divided by the calibration curve slope. 
Electron Microscopy. The microscopy work has been conducted in the "Laboratorio de Microscopias Avanzadas" at "Instituto de Nanociencia de Aragón-Universidad de Zaragoza".

Cryo-TEM. To prepare the sample, a drop of the IONP-ACM suspension was deposited on the carbon side of the copper grid (Electron Microscopy Sciences, Spain) and vitrified by immersion in liquid ethane using an automated device (FEI Vitrobot, The Netherlands). All subsequent manipulations of the grid were carried out in liquid nitrogen, and the TEM images were acquired under cryogenic conditions using a cryo-transfer holder (Gatan Inc., USA). Images were acquired using a Tecnai T20 microscope (FEI Company, The Netherlands) operating at $200 \mathrm{kV}$.

Negative-Stain TEM. For TEM analysis, a carbon-coated 300 mesh copper grid was placed on top of a $5 \mu \mathrm{L}$ droplet of sample (sample facing downward) for $4 \mathrm{~min}$. The grid was then rinsed with a drop of distilled water and subsequently transferred to a $3 \mu \mathrm{L}$ drop of NANO$\mathrm{W}$ ( $2 \%$ in water; Nanoprobes) for $2 \mathrm{~min}$ for negative staining. The grid was quickly rinsed with a drop of water for $1 \mathrm{~min}$ to remove the excess staining solution and dried, contacting the grid edge with filter paper. The grid was stored in an EM grid box under vacuum for future observation by TEM.

EDX Analysis. For electron microscopy analysis, the excised BAT tissue samples were cut into $1 \mathrm{~mm}^{3}$ pieces and fixed with $2.5 \%$ glutaraldehyde/2\% paraformaldehyde in $0.1 \mathrm{M} \mathrm{pH} 7.4$ sodium phosphate buffer for $24 \mathrm{~h}$ at room temperature and subsequently for 5 days at $4{ }^{\circ} \mathrm{C}$. Then, the samples were washed for $30 \mathrm{~min}$ four times with sodium phosphate buffer, postfixed with $2 \%$ osmium, rinsed, dehydrated, and embedded in Durcupan resin (Fluka, Sigma-Aldrich). Semithin sections $(1.5 \mu \mathrm{m})$ were cut with an Ultracut UC-6 ultramicrotome (Leica, Heidelberg, Germany) and stained lightly with $1 \%$ toluidine blue. Finally, ultrathin sections $(0.08 \mu \mathrm{m})$ were cut with a diamond knife, stained with lead citrate (Reynolds solution), and examined under a $300 \mathrm{kV}$ FEI Tecnai F30 transmission electron microscope (FEI Company) equipped with an HAADF detector enabling working in STEM mode for performing EDX analysis.

Brown Adipocyte Culture and Differentiation. Brown preadipocytes were isolated, differentiated, and stained with RedOoil as previously described. ${ }^{74}$

Prussian Blue Staining. Tissue samples were fixed in 4\% PFA for $16 \mathrm{~h}$, dehydrated, and embedded in paraffin. Sections of $5 \mu \mathrm{m}$ were cut and stained with iron stain kit HT20-1KT (Sigma-Aldrich) according to the manufacturer's instructions.

ATP Assay. ATP analysis was performed with ATPlite Luminescence Assay System (PerkinElmer, Waltham, MA, USA) according to the manufacturer's instructions.

RT-qPCR. mRNA was isolated from brown adipocytes using Trizol (ThermoFisher Scientific, Waltham, MA, USA). Reverse transcription was performed with a ProtoScript II First Strand cDNA synthesis kit (New England Biolabs, Ipswitch, MA, USA). qPCR was performed with Power SYBR Green PCR Master Mix (ThermoFisher Scientific).

Data Availability. The raw data that support the findings of this study are available from the corresponding author upon reasonable request.

\section{ASSOCIATED CONTENT}

\section{(s) Supporting Information}

The Supporting Information is available free of charge at https://pubs.acs.org/doi/10.1021/acsnano.0c03229.

$$
\text { Additional information (PDF) }
$$

\section{AUTHOR INFORMATION}

\section{Corresponding Author}

Alexander Pfeifer - Institut für Pharmakologie und Toxikologie, Rheinische Friedrich-Wilhelms-Universität Bonn, 53127 Bonn, Germany; ๑ orcid.org/0000-0001-88056831; Email: alexander.pfeifer@uni-bonn.de

\section{Authors}

Staffan Hildebrand - Institut für Pharmakologie und Toxikologie, Rheinische Friedrich-Wilhelms-Universität Bonn, 53127 Bonn, Germany

Norbert Löwa - 8.23 Metrology for Magnetic Nanoparticles, Physikalisch-Technische Bundesanstalt, 10587 Berlin, Germany

Hendrik Paysen - 8.23 Metrology for Magnetic Nanoparticles, Physikalisch-Technische Bundesanstalt, 10587 Berlin, Germany

Raluca M. Fratila - INMA - Instituto de Nanociencia y Materiales de Aragón (INMA), CSIC-Universidad de Zaragoza, 50009 Zaragoza, Spain; Centro de Investigación Biomédica en Red de Bioingeniería, Biomateriales y Nanomedicina (CIBER-BBN), 28029 Madrid, Spain; - orcid.org/0000-0001-5559-8757

Laia Reverte-Salisa - Institut für Pharmakologie und Toxikologie, Rheinische Friedrich-Wilhelms-Universität Bonn, 53127 Bonn, Germany

Thithawat Trakoolwilaiwan - Biophysics Group, Department of Physics and Astronomy, University College London, London WC1E 6BT, U.K.; UCL Healthcare Biomagnetics Laboratories, London WIS 4BS, U.K.

Zheming Niu - Institut für Pharmakologie und Toxikologie, Rheinische Friedrich-Wilhelms-Universität Bonn, 53127 Bonn, Germany

Georgios Kasparis - Biophysics Group, Department of Physics and Astronomy, University College London, London WC1E 6BT, U.K.; UCL Healthcare Biomagnetics Laboratories, London WIS 4BS, U.K.

Stephanie Franziska Preuss - Institut für Pharmakologie und Toxikologie, Rheinische Friedrich-Wilhelms-Universität Bonn, 53127 Bonn, Germany

Olaf Kosch - 8.23 Metrology for Magnetic Nanoparticles, Physikalisch-Technische Bundesanstalt, 10587 Berlin, Germany

Jesus M. de la Fuente - INMA - Instituto de Nanociencia y Materiales de Aragón (INMA), CSIC-Universidad de Zaragoza, 50009 Zaragoza, Spain; Centro de Investigación Biomédica en Red de Bioingeniería, Biomateriales y Nanomedicina (CIBER-BBN), 28029 Madrid, Spain

Nguyen Thi Kim Thanh - Biophysics Group, Department of Physics and Astronomy, University College London, London WC1E 6BT, U.K.; UCL Healthcare Biomagnetics Laboratories, London WIS 4BS, U.K.; ๑ orcid.org/00000002-4131-5952

Frank Wiekhorst - 8.23 Metrology for Magnetic Nanoparticles, Physikalisch-Technische Bundesanstalt, 10587 Berlin, Germany

Complete contact information is available at: https://pubs.acs.org/10.1021/acsnano.0c03229

\section{Author Contributions}

S.H. and A.P. designed the project. S.H. and S.P. prepared and characterized the artificial chylomicrons. S.H. performed all animal experiments, ATP assays, and qRT-PCRs and was primarily responsible for data collection. N.L. performed all MPS analyses. H.P., O.K., N.L., and F.W. performed all MPI/ MRI analysis and image processing. T.T., G.K., Z.N., and N.T.K.T. synthesized and characterized the zinc-doped MNPs. L.R.S. cultured and differentiated brown adipocytes and performed RedO-oil staining. R.M.F. and J.M.F. performed all TEM imaging and analysis. S.H. and A.P. analyzed the 
results and prepared the manuscript. All authors contributed to the discussion and editing of the manuscript.

\section{Notes}

The authors declare no competing financial interest.

\section{ACKNOWLEDGMENTS}

S.H., L.R.-S., and A.P. thank the DFG (FOR917, 335447717/ SFB1328, and 397484323/SFB TRR 259/1) and DAAD (57172123) for funding. Z.N. was supported by the Bonn International Graduate School of Drug Sciences (BIGS DrugS), University of Bonn, and A.P. is member of the PharmaCenter Bonn, University of Bonn, Germany. N.L., H.P., and F.W. would like to acknowledge the Federal Ministry of Economics and Technology within the TransMeT project "Magnetische Messtechnik für die Größenfraktionierung magnetischer Nanopartikel”, the DFG Grant SHA 1506/2-1 "AMPI: Magnetic particle imaging: Development and evaluation of novel methodology for the assessment of the aorta in vivo in a small animal model of aortic aneurysms", the DFG grant TR 408/9-1 "quantMPI: Establishment of quantitative Magnetic Particle Imaging (MPI) application oriented phantoms for preclinical investigations", and the DFG grant "Matrix in Vision" (SFB 1340/1 2018, no 372486779, project A02). N.T.K.T. thanks EPSRC (EP/M018016/1 and EP/ M015157/1) and AOARD (FA2386-17-1-4042 award) for funding. G.K. would like to acknowledge EPSRC for his Ph.D. studentship. T.T. thanks the Royal Thai Government for his Ph.D. studentship. The microscopy work was conducted in the "Laboratorio de Microscopias Avanzadas" at "Instituto de Nanociencia de Aragón-Universidad de Zaragoza”. The authors acknowledge the LMA-INA for offering access to their instruments and expertise. R.M.F. acknowledges financial support from the European Union's Horizon 2020 research and innovation program under the Marie Skłodowska-Curie grant agreement no. 657215 and from Ministerio de Economía y Competitividad (Ramón y Cajal subprogram RYC-201517640). J.M.F. also acknowledges financial support from Spanish MINECO (project BIO2017-84246-C2-1-R) and DGA and Fondos Feder (Bionanosurf E15_17R). The authors would like to acknowledge the LA-ICPMS laboratory of the Institute of Geosciences and Meterology, University of Bonn, for performing ICP-MS measurements. This article represents contribution number 61 of the LA-ICPMS laboratory.

\section{REFERENCES}

(1) Pfeifer, A.; Hoffmann, L. S. Brown, Beige, and White: The New Color Code of Fat and Its Pharmacological Implications. Annu. Rev. Pharmacol. Toxicol. 2015, 55, 207-27.

(2) Kajimura, S.; Spiegelman, B. M.; Seale, P. Brown and Beige Fat: Physiological Roles Beyond Heat Generation. Cell Metab. 2015, 22 (4), 546-59.

(3) Rosenwald, M.; Wolfrum, C. The Origin and Definition of Brite versus White and Classical Brown Adipocytes. Adipocyte 2014, 3 (1), 4-9.

(4) Cypess, A. M.; Lehman, S.; Williams, G.; Tal, I.; Rodman, D.; Goldfine, A. B.; Kuo, F. C.; Palmer, E. L.; Tseng, Y. H.; Doria, A.; Kolodny, G. M.; Kahn, C. R. Identification and Importance of Brown Adipose Tissue in Adult Humans. N. Engl. J. Med. 2009, 360 (15), 1509-17.

(5) Nedergaard, J.; Bengtsson, T.; Cannon, B. Unexpected Evidence for Active Brown Adipose Tissue in Adult Humans. Am. J. Physiol. Endocrinol. Metab. 2007, 293 (2), E444-52.

(6) Saito, M.; Okamatsu-Ogura, Y.; Matsushita, M.; Watanabe, K.; Yoneshiro, T.; Nio-Kobayashi, J.; Iwanaga, T.; Miyagawa, M.;
Kameya, T.; Nakada, K.; Kawai, Y.; Tsujisaki, M. High Incidence of Metabolically Active Brown Adipose Tissue in Healthy Adult Humans: Effects of Cold Exposure and Adiposity. Diabetes 2009, 58 (7), 1526-31.

(7) van Marken Lichtenbelt, W. D.; Vanhommerig, J. W.; Smulders, N. M.; Drossaerts, J. M.; Kemerink, G. J.; Bouvy, N. D.; Schrauwen, P.; Teule, G. J. Cold-Activated Brown Adipose Tissue in Healthy Men. N. Engl. J. Med. 2009, 360 (15), 1500-8.

(8) Virtanen, K. A.; Lidell, M. E.; Orava, J.; Heglind, M.; Westergren, R.; Niemi, T.; Taittonen, M.; Laine, J.; Savisto, N. J.; Enerback, S.; Nuutila, P. Functional Brown Adipose Tissue in Healthy Adults. N. Engl. J. Med. 2009, 360 (15), 1518-25.

(9) Becher, T.; Palanisamy, S.; Kramer, D. J.; Marx, S. J.; Wibmer, A. G.; Del Gaudio, I.; Butler, S. D.; Jiang, C. S.; Vaughan, R.; Schöder, H.; Di Lorenzo, A.; Mark, A.; Cohen, P. Brown Adipose Tissue Is Associated with Improved Cardiometabolic Health and Regulates Blood Pressure. 2020. bioRxiv. https://www.biorxiv.org/content/ DOI: 10.1101/2020.02.08.933754v2.full (accessed December 4, 2020).

(10) Nedergaard, J.; Bengtsson, T.; Cannon, B. Three Years with Adult Human Brown Adipose Tissue. Ann. N. Y. Acad. Sci. 2010, 1212, E20-36.

(11) Seale, P.; Lazar, M. A. Brown Fat in Humans: Turning up the Heat on Obesity. Diabetes 2009, 58 (7), 1482-1484.

(12) Nedergaard, J.; Bengtsson, T.; Cannon, B. New Powers of Brown Fat: Fighting the Metabolic Syndrome. Cell Metab. 2011, 13 (3), 238-240.

(13) Bartelt, A.; Bruns, O. T.; Reimer, R.; Hohenberg, H.; Ittrich, H.; Peldschus, K.; Kaul, M. G.; Tromsdorf, U. I.; Weller, H.; Waurisch, C.; Eychmuller, A.; Gordts, P. L. S. M.; Rinninger, F.; Bruegelmann, K.; Freund, B.; Nielsen, P.; Merkel, M.; Heeren, J. Brown Adipose Tissue Activity Controls Triglyceride Clearance. Nat. Med. 2011, 17 (2), 200-U93.

(14) Khedoe, P. P. S. J.; Hoeke, G.; Kooijman, S.; Dijk, W.; Buijs, J. T.; Kersten, S.; Havekes, L. M.; Hiemstra, P. S.; Berbee, J. F. P.; Boon, M. R.; Rensen, P. C. N. Brown Adipose Tissue Takes up Plasma Triglycerides Mostly after Lipolysis. J. Lipid Res. 2015, 56 (1), 51-59.

(15) Ouellet, V.; Labbe, S. M.; Blondin, D. P.; Phoenix, S.; Guerin, B.; Haman, F.; Turcotte, E. E.; Richard, D.; Carpentier, A. C. Brown Adipose Tissue Oxidative Metabolism Contributes to Energy Expenditure During Acute Cold Exposure in Humans. J. Clin. Invest. 2012, 122 (2), 545-552.

(16) Townsend, K. L.; Tseng, Y. H. Brown Fat Fuel Utilization and Thermogenesis. Trends Endocrinol. Metab. 2014, 25 (4), 168-77.

(17) Dominiczak, M. H.; Caslake, M. J. Apolipoproteins: Metabolic Role and Clinical Biochemistry Applications. Ann. Clin. Biochem. 2011, 48, 498-515.

(18) Mahley, R. W.; Innerarity, T. L.; Rall, S. C., Jr.; Weisgraber, K. H. Plasma Lipoproteins: Apolipoprotein Structure and Function. J. Lipid Res. 1984, 25 (12), 1277-1294.

(19) Casley-Smith, J. R. The Identification of Chylomicra and Lipoproteins in Tissue Sections and Their Passage into Jejunal Lacteals. J. Cell Biol. 1962, 15, 259-77.

(20) Berbee, J. F. P.; Boon, M. R.; Khedoe, P. P. S. J.; Bartelt, A.; Schlein, C.; Worthmann, A.; Kooijman, S.; Hoeke, G.; Mol, I. M.; John, C.; Jung, C.; Vazirpanah, N.; Brouwers, L. P. J.; Gordts, P. L. S. M.; Esko, J. D.; Hiemstra, P. S.; Havekes, L. M.; Scheja, L.; Heeren, J.; Rensen, P. C. N. Brown Fat Activation Reduces Hypercholesterolaemia and Protects from Atherosclerosis Development. Nat. Commun. 2015, 6.

(21) Mulder, W. J. M.; van Leent, M. M. T.; Lameijer, M.; Fisher, E. A.; Fayad, Z. A.; Perez-Medina, C. High-Density Lipoprotein Nanobiologics for Precision Medicine. Acc. Chem. Res. 2018, 51 (1), 127-137.

(22) Giammanco, A.; Cefalu, A. B.; Noto, D.; Averna, M. R. The Pathophysiology of Intestinal Lipoprotein Production. Front. Physiol. 2015, 6(61).

(23) Goudriaan, J. R.; den Boer, M. A. M.; Rensen, P. C. N.; Febbraio, M.; Kuipers, F.; Romijn, J. A.; Havekes, L. M.; Voshol, P. J. 
Cd36 Deficiency in Mice Impairs Lipoprotein Lipase-Mediated Triglyceride Clearance. J. Lipid Res. 2005, 46 (10), 2175-2181.

(24) Huang, B.; Law, M. W.; Khong, P. L. Whole-Body PET/CT Scanning: Estimation of Radiation Dose and Cancer Risk. Radiology 2009, 251 (1), 166-74.

(25) Blondin, D. P.; Labbe, S. M.; Noll, C.; Kunach, M.; Phoenix, S.; Guerin, B.; Turcotte, E. E.; Haman, F.; Richard, D.; Carpentier, A. C. Selective Impairment of Glucose but Not Fatty Acid or Oxidative Metabolism in Brown Adipose Tissue of Subjects with Type 2 Diabetes. Diabetes 2015, 64 (7), 2388-97.

(26) van Rooijen, B. D.; van der Lans, A. A.; Brans, B.; Wildberger, J. E.; Mottaghy, F. M.; Schrauwen, P.; Backes, W. H.; van Marken Lichtenbelt, W. D. Imaging Cold-Activated Brown Adipose Tissue Using Dynamic T2*-Weighted Magnetic Resonance Imaging and 2Deoxy-2-[18f]Fluoro-D-Glucose Positron Emission Tomography. Invest. Radiol. 2013, 48 (10), 708-14.

(27) Bruns, O. T.; Ittrich, H.; Peldschus, K.; Kaul, M. G.; Tromsdorf, U. I.; Lauterwasser, J.; Nikolic, M. S.; Mollwitz, B.; Merkel, M.; Bigall, N. C.; Sapra, S.; Reimer, R.; Hohenberg, H.; Weller, H.; Eychmuller, A.; Adam, G.; Beisiegel, U.; Heeren, J. RealTime Magnetic Resonance Imaging and Quantification of Lipoprotein Metabolism in Vivo Using Nanocrystals. Nat. Nanotechnol. 2009, 4 (3), 193-201.

(28) Baron, D. M.; Clerte, M.; Brouckaert, P.; Raher, M. J.; Flynn, A. W.; Zhang, H.; Carter, E. A.; Picard, M. H.; Bloch, K. D.; Buys, E. S.; Scherrer-Crosbie, M. In Vivo Noninvasive Characterization of Brown Adipose Tissue Blood Flow by Contrast Ultrasound in Mice. Circ. Cardiovasc. Imaging 2012, 5 (5), 652-9.

(29) Riis-Vestergaard, M. J.; Breining, P.; Pedersen, S. B.; Laustsen, C.; Stodkilde-Jorgensen, H.; Borghammer, P.; Jessen, N.; Richelsen, B. Evaluation of Active Brown Adipose Tissue by the Use of Hyperpolarized [1-(13)C]Pyruvate MRI in Mice. Int. J. Mol. Sci. 2018, 19 (9),

(30) Biederer, S.; Knopp, T.; Sattel, T. F.; Ludtke-Buzug, K.; Gleich, B.; Weizenecker, J.; Borgert, J.; Buzug, T. M. Magnetization Response Spectroscopy of Superparamagnetic Nanoparticles for Magnetic Particle Imaging. J. Phys. D: Appl. Phys. 2009, 42 (20), 205007.

(31) Gleich, B.; Weizenecker, R. Tomographic Imaging Using the Nonlinear Response of Magnetic Particles. Nature 2005, 435 (7046), 1214-1217.

(32) Tay, Z. W.; Chandrasekharan, P.; Zhou, X. Y.; Yu, E.; Zheng, B.; Conolly, S. In Vivo Tracking and Quantification of Inhaled Aerosol Using Magnetic Particle Imaging Towards Inhaled Therapeutic Monitoring. Theranostics 2018, 8 (13), 3676-3687.

(33) Paysen, H.; Loewa, N.; Weber, K.; Kosch, O.; Wells, J.; Schaeffter, T.; Wiekhorst, F. Imaging and Quantification of Magnetic Nanoparticles: Comparison of Magnetic Resonance Imaging and Magnetic Particle Imaging. J. Magn. Magn. Mater. 2019, 475, 382388.

(34) Bauer, L. M.; Situ, S. F.; Griswold, M. A.; Samia, A. C. HighPerformance Iron Oxide Nanoparticles for Magnetic Particle Imaging - Guided Hyperthermia (hMPI). Nanoscale 2016, 8 (24), 12162-9. (35) Tay, Z. W.; Chandrasekharan, P.; Chiu-Lam, A.; Hensley, D. W.; Dhavalikar, R.; Zhou, X. Y.; Yu, E. Y.; Goodwill, P. W.; Zheng, B.; Rinaldi, C.; Conolly, S. M. Magnetic Particle Imaging-Guided Heating in Vivo Using Gradient Fields for Arbitrary Localization of Magnetic Hyperthermia Therapy. ACS Nano 2018, 12 (4), 3699-3713.

(36) Ludewig, P.; Gdaniec, N.; Sedlacik, J.; Forkert, N. D.; Szwargulski, P.; Graeser, M.; Adam, G.; Kaul, M. G.; Krishnan, K. M.; Ferguson, R. M.; Khandhar, A. P.; Walczak, P.; Fiehler, J.; Thomalla, G.; Gerloff, C.; Knopp, T.; Magnus, T. Magnetic Particle Imaging for Real-Time Perfusion Imaging in Acute Stroke. ACS Nano 2017, 11 (10), 10480-10488.

(37) Yu, E. Y.; Chandrasekharan, P.; Berzon, R.; Tay, Z. W.; Zhou, X. Y. Y.; Khandhar, A. P.; Ferguson, R. M.; Kemp, S. J.; Zheng, B.; Goodwill, P. W.; Wendland, M. F.; Krishnan, K. M.; Behr, S.; Carter, J.; Conolly, S. M. Magnetic Particle Imaging for Highly Sensitive, Quantitative, and Safe in Vivo Gut Bleed Detection in a Murine Model. ACS Nano 2017, 11 (12), 12067-12076.
(38) Zheng, B.; von See, M. P.; Yu, E.; Gunel, B.; Lu, K.; Vazin, T.; Schaffer, D. V.; Goodwill, P. W.; Conolly, S. M. Quantitative Magnetic Particle Imaging Monitors the Transplantation, Biodistribution, and Clearance of Stem Cells in Vivo. Theranostics 2016, 6 (3), 291-301.

(39) Krycka, K. L.; Booth, R. A.; Hogg, C. R.; Ijiri, Y.; Borchers, J. A.; Chen, W. C.; Watson, S. M.; Laver, M.; Gentile, T. R.; Dedon, L. R.; Harris, S.; Rhyne, J. J.; Majetich, S. A. Core-Shell Magnetic Morphology of Structurally Uniform Magnetite Nanoparticles. Phys. Rev. Lett. 2010, 104 (20), 207203.

(40) Unni, M.; Uhl, A. M.; Savliwala, S.; Savitzky, B. H.; Dhavalikar, R.; Garraud, N.; Arnold, D. P.; Kourkoutis, L. F.; Andrew, J. S.; Rinaldi, C. Thermal Decomposition Synthesis of Iron Oxide Nanoparticles with Diminished Magnetic Dead Layer by Controlled Addition of Oxygen. ACS Nano 2017, 11 (2), 2284-2303.

(41) Kratz, H.; Taupitz, M.; Ariza de Schellenberger, A.; Kosch, O.; Eberbeck, D.; Wagner, S.; Trahms, L.; Hamm, B.; Schnorr, J. Novel Magnetic Multicore Nanoparticles Designed for MPI and Other Biomedical Applications: From Synthesis to First in Vivo Studies. PLoS One 2018, 13 (1), No. e0190214.

(42) van Dam, A. D.; Nahon, K. J.; Kooijman, S.; van den Berg, S. M.; Kanhai, A. A.; Kikuchi, T.; Heemskerk, M. M.; van Harmelen, V.; Lombes, M.; van den Hoek, A. M.; de Winther, M. P.; Lutgens, E.; Guigas, B.; Rensen, P. C.; Boon, M. R. Salsalate Activates Brown Adipose Tissue in Mice. Diabetes 2015, 64 (5), 1544-54.

(43) Liu, D.; Bordicchia, M.; Zhang, C.; Fang, H.; Wei, W.; Li, J. L.; Guilherme, A.; Guntur, K.; Czech, M. P.; Collins, S. Activation of Mtorc1 Is Essential for Beta-Adrenergic Stimulation of Adipose Browning. J. Clin. Invest. 2016, 126 (5), 1704-16.

(44) Harms, M.; Seale, P. Brown and Beige Fat: Development, Function and Therapeutic Potential. Nat. Med. 2013, 19 (10), 125263.

(45) Blondin, D. P.; Tingelstad, H. C.; Noll, C.; Frisch, F.; Phoenix, S.; Guerin, B.; Turcotte, E. E.; Richard, D.; Haman, F.; Carpentier, A. C. Dietary Fatty Acid Metabolism of Brown Adipose Tissue in ColdAcclimated Men. Nat. Commun. 2017, 814146.

(46) Haman, F.; Peronnet, F.; Kenny, G. P.; Massicotte, D.; Lavoie, C.; Weber, J. M. Partitioning Oxidative Fuels During Cold Exposure in Humans: Muscle Glycogen Becomes Dominant as Shivering Intensifies. J. Physiol. 2005, 566 (1), 247-256.

(47) Fitzgibbons, T. P.; Kogan, S.; Aouadi, M.; Hendricks, G. M.; Straubhaar, J.; Czech, M. P. Similarity of Mouse Perivascular and Brown Adipose Tissues and Their Resistance to Diet-Induced Inflammation. Am. J. Physiol-Heart C 2011, 301 (4), H1425-H1437. (48) Chang, L.; Villacorta, L.; Li, R. X.; Hamblin, M.; Xu, W.; Dou, C. Y.; Zhang, J. F.; Wu, J. R.; Zeng, R.; Chen, Y. E. Loss of Perivascular Adipose Tissue on Peroxisome Proliferator-Activated Receptor-Gamma Deletion in Smooth Muscle Cells Impairs Intravascular Thermoregulation and Enhances Atherosclerosis. Circulation 2012, 126 (9), 1067-U174.

(49) Szczerba, W.; Zukrowski, J.; Przybylski, M.; Sikora, M.; Safonova, O.; Shmeliov, A.; Nicolosi, V.; Schneider, M.; Granath, T.; Oppmann, M.; Strasser, M.; Mandel, K. Pushing up the Magnetisation Values for Iron Oxide Nanoparticles via Zinc Doping: X-Ray Studies on the Particle's Sub-Nano Structure of Different Synthesis Routes. Phys. Chem. Chem. Phys. 2016, 18 (36), 25221-25229.

(50) Vakili-Ghartavol, R.; Momtazi-Borojeni, A. A.; VakiliGhartavol, Z.; Aiyelabegan, H. T.; Jaafari, M. R.; Rezayat, S. M.; Arbabi Bidgoli, S. Toxicity Assessment of Superparamagnetic Iron Oxide Nanoparticles in Different Tissues. Artif. Cells, Nanomed., Biotechnol. 2020, 48 (1), 443-451.

(51) Plum, L. M.; Rink, L.; Haase, H. The Essential Toxin: Impact of Zinc on Human Health. Int. J. Environ. Res. Public Health 2010, 7 (4), 1342-65.

(52) Zhu, S.; Xu, X.; Rong, R.; Li, B.; Wang, X. Evaluation of ZincDoped Magnetite Nanoparticle Toxicity in the Liver and Kidney of Mice after Sub-Chronic Intragastric Administration. Toxicol. Res. (Cambridge, U. K.) 2016, 5 (1), 97-106. 
(53) Lee, J.; Yu, W. J.; Song, J.; Sung, C.; Jeong, E. J.; Han, J. S.; Kim, P.; Jo, E.; Eom, I.; Kim, H. M.; Kwon, J. T.; Choi, K.; Choi, J.; Kim, H.; Lee, H.; Park, J.; Jin, S. M.; Park, K. Developmental Toxicity of Intravenously Injected Zinc Oxide Nanoparticles in Rats. Arch. Pharmacal Res. 2016, 39 (12), 1682-1692.

(54) Geiser, J.; Venken, K. J.; De Lisle, R. C.; Andrews, G. K. A Mouse Model of Acrodermatitis Enteropathica: Loss of Intestine Zinc Transporter Zip4 (Slc39a4) Disrupts the Stem Cell Niche and Intestine Integrity. PLoS Genet. 2012, 8 (6), No. e1002766.

(55) Galvez-Peralta, M.; He, L.; Jorge-Nebert, L. F.; Wang, B.; Miller, M. L.; Eppert, B. L.; Afton, S.; Nebert, D. W. Zip8 Zinc Transporter: Indispensable Role for Both Multiple-Organ Organogenesis and Hematopoiesis in Utero. PLoS One 2012, 7 (5), No. e36055.

(56) Weber, A.; Werner, F.; Weizenecker, J.; Buzug, T. M.; Knopp, T. Artifact Free Reconstruction with the System Matrix Approach by Overscanning the Field-Free-Point Trajectory in Magnetic Particle Imaging. Phys. Med. Biol. 2016, 61 (2), 475-87.

(57) Knopp, T.; Them, K.; Kaul, M.; Gdaniec, N. Joint Reconstruction of Non-Overlapping Magnetic Particle Imaging Focus-Field Data. Phys. Med. Biol. 2015, 60 (8), L15-21.

(58) Chandrasekharan, P.; Tay, Z. W.; Zhou, X. Y.; Yu, E.; Orendorff, R.; Hensley, D.; Huynh, Q.; Fung, K. L. B.; VanHook, C. C.; Goodwill, P.; Zheng, B.; Conolly, S. A Perspective on a Rapid and Radiation-Free Tracer Imaging Modality, Magnetic Particle Imaging, with Promise for Clinical Translation. Br. J. Radiol. 2018, 91 (1091), 20180326.

(59) Tay, Z. W.; Hensley, D. W.; Chandrasekharan, P.; Zheng, B.; Conolly, S. M. Optimization of Drive Parameters for Resolution, Sensitivity and Safety in Magnetic Particle Imaging. IEEE Trans. Med. Imaging 2020, 39 (5), 1724-1734.

(60) Graeser, M.; Thieben, F.; Szwargulski, P.; Werner, F.; Gdaniec, N.; Boberg, M.; Griese, F.; Moddel, M.; Ludewig, P.; van de Ven, D.; Weber, O. M.; Woywode, O.; Gleich, B.; Knopp, T. Human-Sized Magnetic Particle Imaging for Brain Applications. Nat. Commun. 2019, 10 (1), 1936.

(61) Carneiro, M. M.; Miname, M. H.; Gagliardi, A. C.; Pereira, C.; Pereira, A. C.; Krieger, J. E.; Maranhao, R. C.; Santos, R. D. The Removal from Plasma of Chylomicrons and Remnants Is Reduced in Heterozygous Familial Hypercholesterolemia Subjects with Identified LDL Receptor Mutations: Study with Artificial Emulsions. Atherosclerosis 2012, 221 (1), 268-274.

(62) Meyer, J. M.; Ji, A.; Cai, L.; van der Westhuyzen, D. R. HighCapacity Selective Uptake of Cholesteryl Ester from Native LDL During Macrophage Foam Cell Formation. J. Lipid Res. 2012, 53 (10), 2081-91.

(63) Shadid, S.; Koutsari, C.; Jensen, M. D. Direct Free Fatty Acid Uptake into Human Adipocytes in Vivo: Relation to Body Fat Distribution. Diabetes 2007, 56 (5), 1369-75.

(64) Hachani, R.; Lowdell, M.; Birchall, M.; Hervault, A.; Mertz, D.; Begin-Coline, S.; Thanh, N. T. K. Polyol Synthesis, Functionalisation, and Biocompatibility Studies of Superparamagnetic Iron Oxide Nanoparticles as Potential MRI Contrast Agents. Nanoscale 2016, 8 (6), 3278-3287.

(65) Loewa, N.; Wiekhorst, F.; Gemeinhardt, I.; Ebert, M.; Schnorr, J.; Wagner, S.; Taupitz, M.; Trahms, L. Cellular Uptake of Magnetic Nanoparticles Quantified by Magnetic Particle Spectroscopy. IEEE Trans. Magn. 2013, 49 (1), 275-278.

(66) Poller, W. C.; Lowa, N.; Wiekhorst, F.; Taupitz, M.; Wagner, S.; Moller, K.; Baumann, G.; Stangl, V.; Trahms, L.; Ludwig, A. Magnetic Particle Spectroscopy Reveals Dynamic Changes in the Magnetic Behavior of Very Small Superparamagnetic Iron Oxide Nanoparticles During Cellular Uptake and Enables Determination of Cell-Labeling Efficacy. J. Biomed. Nanotechnol. 2016, 12 (2), 337-46.

(67) Löwa, N.; Seidel, M.; Radon, P.; Wiekhorst, F. Magnetic Nanoparticles in Different Biological Environments Analyzed by Magnetic Particle Spectroscopy. J. Magn. Magn. Mater. 2017, 427, 133-138.
(68) Paysen, H.; Wells, J.; Kosch, O.; Steinhoff, U.; Franke, J.; Trahms, L.; Schaeffter, T.; Wiekhorst, F. Improved Sensitivity and Limit-of-Detection Using a Receive-Only Coil in Magnetic Particle Imaging. Phys. Med. Biol. 2018, 63 (13),13NT02.

(69) Rahmer, J.; Weizenecker, J.; Gleich, B.; Borgert, J. Signal Encoding in Magnetic Particle Imaging: Properties of the System Function. BMC Med. Imaging 2009, 9 (4) DOI: 10.1186/1471-23429-4.

(70) Rahmer, J.; Weizenecker, J.; Gleich, B.; Borgert, J. Analysis of a 3-D System Function Measured for Magnetic Particle Imaging. IEEE Trans. Med. Imaging 2012, 31 (6), 1289-99.

(71) Marks, G.; Steinhoff, U.; Wiekhorst, F. A Model for Uncertainty Influences on Static Magnetisation Measurements on Magnetic Nanoparticles. Journal of Physics: Conference Series 2018, 1065 (072030).

(72) Ludwig, A.; Poller, W. C.; Westphal, K.; Minkwitz, S.; LattigTunnemann, G.; Metzkow, S.; Stangl, K.; Baumann, G.; Taupitz, M.; Wagner, S.; Schnorr, J.; Stangl, V. Rapid Binding of Electrostatically Stabilized Iron Oxide Nanoparticles to Thp-1 Monocytic Cells via Interaction with Glycosaminoglycans. Basic Res. Cardiol. 2013, 108 (2).

(73) Chantrell, R.; Popplewell, J.; Charles, S. Measurements of Particle Size Distribution Parameters in Ferrofluids. IEEE Trans. Magn. 1978, 14 (5), 975-977.

(74) Haas, B.; Mayer, P.; Jennissen, K.; Scholz, D.; Berriel Diaz, M.; Bloch, W.; Herzig, S.; Fassler, R.; Pfeifer, A. Protein Kinase G Controls Brown Fat Cell Differentiation and Mitochondrial Biogenesis. Sci. Signal 2009, 2 (99), No. ra78. 